\title{
STOCHASTIC PARTIAL DIFFERENTIAL EQUATIONS DRIVEN BY PURELY SPATIAL NOISE
}

\author{
S. V. LOTOTSKY AND B. L. ROZOVSKII
}

\begin{abstract}
We study bilinear stochastic parabolic and elliptic PDEs driven by purely spatial white noise. Even the simplest equations driven by this noise often do not have a square-integrable solution and must be solved in special weighted spaces. We demonstrate that the Cameron-Martin version of the Wiener chaos decomposition is an effective tool to study both stationary and evolution equations driven by space-only noise. The paper presents results about solvability of such equations in weighted Wiener chaos spaces and studies the long-time behavior of the solutions of evolution equations with space-only noise.
\end{abstract}

July 15, 2018

\section{INTRODUCTION}

Stochastic PDEs of the form

$$
\dot{u}(t, x)=\mathbf{A} u(t, x)+\mathbf{M} u(t, x) \cdot \dot{W}(t, x),
$$

where $\mathbf{A}$ and $\mathbf{M}$ are linear partial differential operators and $\dot{W}(t, x)$ is space-time noise, are usually referred to as bilinear evolution SPDEs.1 These equations are of interest in various applications: nonlinear filtering for hidden Markov models [30, propagation of magnetic field in random flow [2] stochastic transport [6, 7, 20]), porous media [3] and others. The theory and the applications of bilinear SPDEs have been actively investigated for a few decades now; see, for example, [4, 13, 23, 27, 28, 29, etc.].

In contrast, very little is known about bilinear parabolic and elliptic equations driven by purely spatial Gaussian white noise $\dot{W}(x)$. Important examples of these equations include:

2000 Mathematics Subject Classification. Primary 60H15; Secondary 35R60, 60H40.

Key words and phrases. Generalized Random Elements, Malliavin Calculus, Skorokhod Integral, Wiener Chaos, Weighted Spaces.

S. V. Lototsky acknowledges support from the Sloan Research Fellowship and the NSF CAREER award DMS-0237724.

B. L. Rozovskii acknowledges support from NSF Grant DMS 0604863, ARO Grant W911NF-071-0044, and ONR Grant N00014-07-1-0044.

An earlier version of the paper was posted on Archives http://arxiv.org/abs/math.PR/0505551, May 25, 2005.

${ }^{1}$ Bilinear SPDEs differ from linear by the term including multiplicative noise. Bilinear SPDEs are technically more difficult then linear. On the other hand, multiplicative models preserve many features of the unperturbed equation, such as positivity of the solution and conservation of mass, and are often more "physical". 
1. Heat equation with random potential modeled by spatial white noise:

$$
\dot{u}(t, x)=\Delta u(t, x)+u(t, x) \diamond \dot{W}(x),
$$

where $\diamond$ denotes the Wick product, which, in this case, coincides with the Skorokhod integral in the sense of Malliavin calculus. A surprising discovery made in [9] was that the spatial regularity of the solution of equation (1.2) is better than in the case of similar equation driven by the space-time white noise.

2. Stochastic Poisson equations in random medium:

$$
\nabla\left(A_{\varepsilon}(x) \diamond \nabla u(x)\right)=f(x),
$$

where $A_{\varepsilon}(x):=(a(x)+\varepsilon \dot{W}(x)), a(x)$ is a deterministic positive-definite matrix, and $\varepsilon$ is a positive number. (Note that $a(x) \diamond \nabla u(x)=a(x) \nabla u(x)$ ).

3. Heat equation in random medium:

$$
\dot{v}(t, x)=\nabla\left(A_{\varepsilon}(x) \diamond \nabla v(t, x)\right)+g(t, x) .
$$

Note that the matrix $A_{\varepsilon}$ in equations (1.3) and (1.4) is not necessarily positive definite; only its expectation $a(x)$ is.

Equations (1.3) and (1.4) are random perturbation of the deterministic Poisson and Heat equations. An important feature of these type of perturbation is that the resulting equations are unbiased in that they preserve the mean dynamics: the functions $u_{0}(x):=\mathbb{E} u(x)$ and $v_{0}:=\mathbb{E} v(t, x)$ solve the deterministic Poisson equation,

$$
\nabla\left(a(x) \nabla u_{0}(t, x)\right)=\mathbb{E} f(x)
$$

and the deterministic Heat equation

$$
\dot{v}_{0}(t, x)=\nabla\left(a(x) v_{0}(t, x)\right)+\mathbb{E} g(t, x),
$$

respectively.

The objective of this paper is to develop a systematic approach to bilinear SPDEs driven by purely spatial Gaussian noise. More specifically, we will investigate bilinear parabolic equations

$$
\frac{\partial v(t, x)}{\partial t}=\mathbf{A} v(t, x)+\mathbf{M} v(t, x) \diamond \dot{W}(x)-f(x)
$$

and elliptic equations

$$
\mathbf{A} u(x)+\mathbf{M} u(x) \diamond \dot{W}(x)=f(x) .
$$

for a wide range of operators $\mathbf{A}$ and $\mathbf{M}$.

Purely spatial white noise is an important type of stationary perturbations. However, except for elliptic equations with additive random forcing [5, 22, 26, SPDEs driven by spatial noise have not been investigated nearly as extensively as those driven by strictly temporal or space-time noise.

In the case of spatial white noise, there is no natural and convenient filtration, especially in the dimension $d>2$. Therefore, it makes sense to consider anticipative solutions. This rules out Itô calculus and makes it necessary to rely on more nuanced techniques provided by Malliavin calculus. 
In this paper, we deal with broad classes of operators $\mathbf{A}$ and $\mathbf{M}$ that were investigated previously for causal (nonanticipating) solutions of the equation (1.1) driven by spacetime white noise.

The notion of ellipticity for SPDEs is more restrictive then in deterministic theory. Traditionally, nonanticipating solutions of equation (1.1) were studied under the following assumptions:

(i) The operator $\mathbf{A}-\frac{1}{2} \mathbf{M M}^{\star}$ is "elliptic" (possibly degenerate coercive) operator.

Of course, this assumption does not hold for equation (1.4) and other equations in which the operators $\mathbf{A}$ and $\mathbf{M}$ have the same order. Therefore, it is important to study equations (1.6) and (1.5) under weaker assumptions, for example

(ii) The operator $\mathbf{A}$ is coercive and ord $(\mathbf{M}) \leq \operatorname{ord}(\mathbf{A})$.

In 1981, it was shown by Krylov and Rozovskii [15] that, unless assumption (i) holds, equation (1.1) has no solutions in the space $L_{2}(\Omega ; X)$ of square integrable (in probability) solutions in any reasonable functional space $X$. The same effect holds for bilinear SPDEs driven by space only white noise.

Numerous attempts to investigate solutions of stochastic PDEs violating the stochastic ellipticity conditions were made since then. In particular it was shown in [19, 21, 25] that if the operator $\mathbf{A}$ is coercive ("elliptic") and ord (M) is strictly less then $\operatorname{ord}(\mathbf{A})$ then there there exists a unique generalized (Wiener Chaos) nonanticipative solution of equation (1.1). This generalized solution is a formal Wiener Chaos series $u=\sum_{|\alpha|<\infty} u_{\alpha} \xi_{\alpha}$, where $\left\{\xi_{\alpha}\right\}_{|\alpha|<\infty}$ is the Cameron-Martin orthonormal basis in the space $L_{2}(\Omega)$. Regularity of this solution is determined by system of positive weights $\left\{r_{\alpha}\right\}_{|\alpha|<\infty}$ and a function space $X$ such that

$$
\|u\|_{\mathcal{R}, X}^{2}:=\sum_{|\alpha|<\infty} r_{\alpha}^{2}\left\|u_{\alpha}\right\|_{L_{2}((0, T) ; X)}^{2}<\infty .
$$

The stochastic Fourier coefficients $u_{\alpha}$ satisfy a lower-triangular system of deterministic PDEs. This system, called propagator, is uniquely determined by the underlying equation (1.1).

Stochastic spaces equipped with the norms similar to (1.7) have been known for quite some time; see, e.g. [11, 12, 25]. For historical remarks regarding other types of generalized solutions and applications to SPDEs see the review paper [18] and the references therein.

The Wiener Chaos is a bona fide generalization of the classical Itô solution: if exists, a non-antisipating square integrable Itô solution coincides with the Wiener Chaos solution.

In this paper, we establish existence and uniqueness of Wiener Chaos solutions for stationary (elliptic) equations of the type (1.6) and evolution (parabolic) equations of the type (1.5). These results are proved under assumption (ii) that allows us in particular to deal with equations like (1.3) and (1.4). In many cases we are able to find optimal or near-optimal systems of weights $\left\{r_{\alpha}\right\}_{|\alpha|<\infty}$ that guarantee (1.7) 
Finally, we establish the convergence, as $t \rightarrow+\infty$, of the solution of the evolution equation to the solution of the related stationary equation.

The structure of the paper is as follows. Section 2 reviews the definition of the Skorokhod integral in the framework of the Malliavin calculus and shows how to compute the integral using Wiener chaos. Sections 3 and 4 deal with existence and uniqueness of solutions to abstract evolution and stationary equations, respectively, driven by a general (not necessarily white) spatial Gaussian noise; Section 4 describes also the limiting behavior of the solution of the evolution equation; Section 5 illustrates the general results for bilinear SPDEs driven by purely spatial white noise.

\section{Weighted Wiener Chaos and Malliavin Calculus}

Let $\mathbb{F}=(\Omega, \mathcal{F}, \mathbb{P})$ be a complete probability space, and $\mathcal{U}$, a real separable Hilbert space with inner product $(\cdot, \cdot) \mathcal{U}$. On $\mathbb{F}$, consider a zero-mean Gaussian family

$$
\dot{W}=\{\dot{W}(h), h \in \mathcal{U}\}
$$

so that

$$
\mathbb{E}\left(\dot{W}\left(h_{1}\right) \dot{W}\left(h_{2}\right)\right)=\left(h_{1}, h_{2}\right)_{\mathcal{U}}
$$

It suffice, for our purposes, to assume that $\mathcal{F}$ is the $\sigma$-algebra generated by $\dot{W}$. Given a real separable Hilbert space $X$, we denote by $L_{2}(\mathbb{F} ; X)$ the Hilbert space of squareintegrable $\mathcal{F}$-measurable $X$-valued random elements $f$. In particular,

$$
(f, g)_{L_{2}(\mathbb{F} ; X)}^{2}:=\mathbb{E}(f, g)_{X}^{2} .
$$

When $X=\mathbb{R}$, we write $L_{2}(\mathbb{F})$ instead of $L_{2}(\mathbb{F} ; \mathbb{R})$.

Definition 2.1. A formal series

$$
\dot{W}=\sum_{k} \dot{W}\left(\mathfrak{u}_{k}\right) \mathfrak{u}_{k}
$$

where $\left\{\mathfrak{u}_{k}, k \geq 1\right\}$ is a complete orthonormal basis in $\mathcal{U}$, is called (Gaussian) white noise on $\mathcal{U}$.

The white noise on $\mathcal{U}=L_{2}(G)$, where $G$ is a domain in $\mathbb{R}^{d}$, is usually referred to as a spatial or space white noise (on $\left.L_{2}(G)\right)$. The space white noise is of central importance for this paper.

Below, we will introduce a class of spaces that are convenient for treating nonlinear functionals of white noise, in particular, solutions of SPDEs driven by white noise.

Given an orthonormal basis $\mathfrak{U}=\left\{\mathfrak{u}_{k}, k \geq 1\right\}$ in $\mathcal{U}$, define a collection $\left\{\xi_{k}, k \geq 1\right\}$ of independent standard Gaussian random variables so that $\xi_{k}=\dot{W}\left(\mathfrak{u}_{k}\right)$. Denote by $\mathcal{J}$ the collection of multi-indices $\alpha$ with $\alpha=\left(\alpha_{1}, \alpha_{2}, \ldots\right)$ so that each $\alpha_{k}$ is a non-negative integer and $|\alpha|:=\sum_{k \geq 1} \alpha_{k}<\infty$. For $\alpha, \beta \in \mathcal{J}$, we define

$$
\alpha+\beta=\left(\alpha_{1}+\beta_{1}, \alpha_{2}+\beta_{2}, \ldots\right), \quad \alpha !=\prod_{k \geq 1} \alpha_{k} !
$$


By $(0)$ we denote the multi-index with all zeroes. By $\varepsilon_{i}$ we denote the multi-index $\alpha$ with $\alpha_{i}=1$ and $\alpha_{j}=0$ for $j \neq i$. With this notation, $n \varepsilon_{i}$ is the multi-index $\alpha$ with $\alpha_{i}=n$ and $\alpha_{j}=0$ for $j \neq i$. The following inequality holds (see Appendix for the proof):

$$
|\alpha| ! \leq \alpha !(2 \mathbb{N})^{2 \alpha}, \quad \text { where }(2 \mathbb{N})^{2 \alpha}=\prod_{k \geq 1}(2 k)^{2 \alpha_{k}}
$$

Define the collection of random variables $\Xi=\left\{\xi_{\alpha}, \alpha \in \mathcal{J}\right\}$ as follows:

$$
\xi_{\alpha}=\prod_{k}\left(\frac{H_{\alpha_{k}}\left(\xi_{k}\right)}{\sqrt{\alpha_{k} !}}\right),
$$

where

$$
H_{n}(x)=(-1)^{n} e^{x^{2} / 2} \frac{d^{n}}{d x^{n}} e^{-x^{2} / 2}
$$

is Hermite polynomial of order $n$.

Theorem 2.2 (Cameron and Martin [1]). The collection $\Xi=\left\{\xi_{\alpha}, \alpha \in \mathcal{J}\right\}$ is an orthonormal basis in $L_{2}(\mathbb{F})$ : if $\eta \in L_{2}(\mathbb{F})$ and $\eta_{\alpha}=\mathbb{E}\left(\eta \xi_{\alpha}\right)$, then $\eta=\sum_{\alpha \in \mathcal{J}} \eta_{\alpha} \xi_{\alpha}$ and $\mathbb{E}|\eta|^{2}=\sum_{\alpha \in \mathcal{J}} \eta_{\alpha}^{2}$

Expansions with respect to the Cameron-Martin basis $\Xi$ is usually referred to as Wiener Chaos. Next, we introduce a modification of the Wiener Chaos expansion which will be called weighted Wiener Chaos.

Let $\mathcal{R}$ be a bounded linear operator on $L_{2}(\mathbb{F})$ defined by $\mathcal{R} \xi_{\alpha}=r_{\alpha} \xi_{\alpha}$ for every $\alpha \in \mathcal{J}$, where the weights $\left\{r_{\alpha}, \alpha \in \mathcal{J}\right\}$ are positive numbers. By Theorem 2.2 , $\mathcal{R}$ is bounded if and only if the weights $r_{\alpha}$ are uniformly bounded from above: $r_{\alpha}<C$ for all $\alpha \in \mathcal{J}$, with $C$ independent of $\alpha$. The inverse operator $\mathcal{R}^{-1}$ is defined by $\mathcal{R}^{-1} \xi_{\alpha}=r_{\alpha}^{-1} \xi_{\alpha}$.

We extend $\mathcal{R}$ to an operator on $L_{2}(\mathbb{F} ; X)$ by defining $\mathcal{R} f$ as the unique element of $L_{2}(\mathbb{F} ; X)$ so that, for all $g \in L_{2}(\mathbb{F} ; X)$,

$$
\mathbb{E}(\mathcal{R} f, g)_{X}=\sum_{\alpha \in \mathcal{J}} r_{\alpha} \mathbb{E}\left((f, g)_{X} \xi_{\alpha}\right)
$$

Denote by $\mathcal{R} L_{2}(\mathbb{F} ; X)$ the closure of $L_{2}(\mathbb{F} ; X)$ with respect to the norm

$$
\|f\|_{\mathcal{R} L_{2}(\mathbb{F} ; X)}^{2}:=\|\mathcal{R} f\|_{L_{2}(\mathbb{F} ; X)}^{2} .
$$

Then the elements of $\mathcal{R} L_{2}(\mathbb{F} ; X)$ can be identified with a formal series $\sum_{\alpha \in \mathcal{J}} f_{\alpha} \xi_{\alpha}$, where $f_{\alpha} \in X$ and $\sum_{\alpha \in \mathcal{J}}\left\|f_{\alpha}\right\|_{X}^{2} r_{\alpha}^{2}<\infty$.

We define the space $\mathcal{R}^{-1} L_{2}(\mathbb{F} ; X)$ as the dual of $\mathcal{R} L_{2}(\mathbb{F} ; X)$ relative to the inner product in the space $L_{2}(\mathbb{R} ; X)$ :

$$
\mathcal{R}^{-1} L_{2}(\mathbb{F} ; X)=\left\{g \in L_{2}(\mathbb{F} ; X): \mathcal{R}^{-1} g \in L_{2}(\mathbb{F} ; X)\right\} .
$$

For $f \in \mathcal{R} L_{2}(\mathbb{F} ; X)$ and $g \in \mathcal{R}^{-1} L_{2}(\mathbb{F})$ we define the scalar product

$$
\langle\langle f, g\rangle\rangle:=\mathbb{E}\left((\mathcal{R} f)\left(\mathcal{R}^{-1} g\right)\right) \in X
$$


In what follows, we will identify the operator $\mathcal{R}$ with the corresponding collection $\left(r_{\alpha}, \alpha \in \mathcal{J}\right)$. Note that if $u \in \mathcal{R}_{1} L_{2}(\mathbb{F} ; X)$ and $v \in \mathcal{R}_{2} L_{2}(\mathbb{F} ; X)$, then both $u$ and $v$ belong to $\mathcal{R} L_{2}(\mathbb{F} ; X)$, where $r_{\alpha}=\min \left(r_{1, \alpha}, r_{2, \alpha}\right)$. As usual, the argument $X$ will be omitted if $X=\mathbb{R}$.

Important particular cases of the space $\mathcal{R} L_{2}(\mathbb{F} ; X)$ correspond to the following weights:

$$
r_{\alpha}^{2}=\prod_{k=1}^{\infty} q_{k}^{\alpha_{k}}
$$

where $\left\{q_{k}, k \geq 1\right\}$ is a non-increasing sequence of positive numbers with $q_{1} \leq 1$ (see [19, 25]);

$$
r_{\alpha}^{2}=(\alpha !)^{\rho}(2 \mathbb{N})^{\ell \alpha}, \rho \leq 0, \ell \leq 0, \quad \text { where }(2 \mathbb{N})^{\ell \alpha}=\prod_{k \geq 1}(2 k)^{\ell \alpha_{k}}
$$

This set of weights defines Kondratiev's spaces $(\mathcal{S})_{\rho, \ell}(X)$.

Now we will sketch the basics of Malliavin calculus on $\mathcal{R} L_{2}(\mathbb{F} ; X)$.

Denote by $\mathbf{D}$ the Malliavin derivative on $L_{2}(\mathbb{F})$ (see e.g. [24]). In particular, if $F: \mathbb{R}^{N} \rightarrow \mathbb{R}$ is a smooth function and $h_{i} \in \mathcal{U}, i=1, \ldots N$, then

$$
\mathbf{D} F\left(\dot{W}\left(h_{1}\right), \ldots \dot{W}\left(h_{N}\right)\right)=\sum_{i=1}^{N} \frac{\partial F}{\partial x_{i}}\left(\dot{W}\left(h_{1}\right), \ldots, \dot{W}\left(h_{N}\right)\right) h_{i} \in L_{2}(\mathbb{F} ; \mathcal{U}) .
$$

It is known 24] that the domain $\mathbb{D}^{1,2}(\mathbb{F})$ of the operator $\mathbf{D}$ is a dense linear subspace of $L_{2}(\mathbb{F})$.

The adjoint of the Malliavin derivative on $L_{2}(\mathbb{F})$ is the Itô-Skorokhod integral and is traditionally denoted by $\delta$ [24]. We will keep this notation for the extension of this operator to $\mathcal{R} L_{2}(\mathbb{F} ; X \otimes \mathcal{U})$.

For $f \in \mathcal{R} L_{2}(\mathbb{F} ; X \otimes \mathcal{U})$, we define $\delta(f)$ as the unique element of $\mathcal{R} L_{2}(\mathbb{F} ; X)$ with the property

$$
\left\langle\langle\delta(f), \varphi\rangle=\mathbb{E}\left(\mathcal{R} f, \mathcal{R}^{-1} \mathbf{D} \varphi\right)_{\mathcal{U}}\right.
$$

for every $\varphi$ satisfying $\varphi \in \mathcal{R}^{-1} L_{2}(\mathbb{F})$ and $\mathbf{D} \varphi \in \mathcal{R}^{-1} L_{2}(\mathbb{F} ; \mathcal{U})$.

Next, we derive the expressions for the Malliavin derivative $\mathbf{D}$ and its adjoint $\delta$ in the basis $\Xi$. To begin, we compute $\mathbf{D}\left(\xi_{\alpha}\right)$.

Proposition 2.3. For each $\alpha \in \mathcal{J}$, we have

$$
\mathbf{D}\left(\xi_{\alpha}\right)=\sum_{k \geq 1} \sqrt{\alpha_{k}} \xi_{\alpha-\varepsilon_{k}} \mathfrak{u}_{k}
$$

Proof. The result follows by direct computation using the property (2.7) of the Malliavin derivative and the relation $H_{n}^{\prime}(x)=n H_{n-1}(x)$ for the Hermite polynomials (cf. [24]). 
Obviously, the set $\mathcal{J}$ is not invariant with respect to substraction. In particular, the expression $\alpha-\varepsilon_{k}$ is undefined if $\alpha_{k}=0$. In (2.9) and everywhere below in this paper where undefined expressions of this type appear, we use the following convention: if $\alpha_{k}=0$, then $\sqrt{\alpha_{k}} \xi_{\alpha-\varepsilon_{k}}=0$.

Proposition 2.4. For $\xi_{\alpha} \in \Xi, h \in X$, and $\mathfrak{u}_{k} \in \mathfrak{U}$, we have

$$
\delta\left(\xi_{\alpha} h \otimes \mathfrak{u}_{k}\right)=h \sqrt{\alpha_{k}+1} \xi_{\alpha+\varepsilon_{k}} .
$$

Proof. It is enough to verify (2.8) with $f=h \otimes \mathfrak{u}_{k} \xi_{\alpha}$ and $\varphi=\xi_{\beta}$, where $h \in X$. By (2.9),

$$
\mathbb{E}(f, \mathbf{D} \varphi)_{\mathcal{U}}=\sqrt{\beta_{k}} h \mathbb{E}\left(\xi_{\alpha} \xi_{\beta-\varepsilon_{k}}\right)= \begin{cases}\sqrt{\alpha_{k}+1} h, & \text { if } \alpha=\beta-\varepsilon_{k}, \\ 0, & \text { if } \alpha \neq \beta-\varepsilon_{k}\end{cases}
$$

In other words,

$$
\mathbb{E}\left(\xi_{\alpha} h \otimes \mathfrak{u}_{k}, \mathbf{D} \xi_{\beta}\right)_{\mathcal{U}}=h \mathbb{E}\left(\sqrt{\alpha_{k}+1} \xi_{\alpha+\varepsilon_{k}} \xi_{\beta}\right)
$$

for all $\beta \in \mathcal{J}$.

Remark 2.5. The operator $\delta \mathbf{D}$ is linear and unbounded on $L_{2}(\mathbb{F})$; it follows from Propositions 2.3 and 2.4 that the random variables $\xi_{\alpha}$ are eigenfunctions of this operator:

$$
\delta\left(\mathbf{D}\left(\xi_{\alpha}\right)\right)=|\alpha| \xi_{\alpha}
$$

To give an alternative characterization of the operator $\delta$, we define a new operation on the elements of $\Xi$.

Definition 2.6. For $\xi_{\alpha}, \xi_{\beta}$ from $\Xi$, define the Wick product

$$
\xi_{\alpha} \diamond \xi_{\beta}:=\sqrt{\left(\frac{(\alpha+\beta) !}{\alpha ! \beta !}\right)} \xi_{\alpha+\beta} .
$$

In particular, taking in (2.6) $\alpha=k \varepsilon_{i}$ and $\beta=n \varepsilon_{i}$, and using (2.3), we get

$$
H_{k}\left(\xi_{i}\right) \diamond H_{n}\left(\xi_{i}\right)=H_{k+n}\left(\xi_{i}\right) .
$$

By linearity, we define the Wick product $f \diamond \eta$ for $f \in \mathcal{R} L_{2}(\mathbb{F} ; X)$ and $\eta \in \mathcal{R} L_{2}(\mathbb{F})$ : if $f=\sum_{\alpha \in \mathcal{J}} f_{\alpha} \xi_{\alpha}, f_{\alpha} \in X$, and $\eta=\sum_{\alpha \in \mathcal{J}} \eta_{\alpha} \xi_{\alpha}, \eta_{\alpha} \in \mathbb{R}$, then

$$
f \diamond \eta=\sum_{\alpha, \beta} f_{\alpha} \eta_{\beta} \xi_{\alpha} \diamond \xi_{\beta}
$$

Proposition 2.7. If $f \in \mathcal{R} L_{2}(\mathbb{F} ; X)$ and $\eta \in \mathcal{R} L_{2}(\mathbb{F})$, then $f \diamond \eta$ is an element of $\overline{\mathcal{R}} L_{2}(\mathbb{F} ; X)$ for a suitable operator $\overline{\mathcal{R}}$.

Proof. It follows from (2.6) that $f \diamond \eta=\sum_{\alpha \in \mathcal{J}} F_{\alpha} \xi_{\alpha}$ and

$$
F_{\alpha}=\sum_{\beta, \gamma \in \mathcal{J}: \beta+\gamma=\alpha} \sqrt{\left(\frac{(\beta+\gamma) !}{\beta ! \gamma !}\right)} f_{\beta} \eta_{\gamma}
$$


Therefore, each $F_{\alpha} X$ is an element of $X$, because, for every $\alpha \in \mathcal{J}$, there are only finitely many multi-indices $\beta, \gamma$ satisfying $\beta+\gamma=\alpha$. It is known [18, Proposition 7.1] that

$$
\sum_{\alpha \in \mathcal{J}}(2 \mathbb{N})^{q \alpha}<\infty \text { if and only if } q<-1 .
$$

Therefore, $f \diamond \eta \in \overline{\mathcal{R}} L_{2}(\mathbb{F} ; X)$, where the operator $\overline{\mathcal{R}}$ can be defined using the weights $\bar{r}_{\alpha}^{2}=(2 \mathbb{N})^{-2 \alpha} /\left(1+\left\|F_{\alpha}\right\|_{X}^{2}\right)$.

An immediate consequence of Proposition 2.4 and Definition 2.6 is the following identity:

$$
\delta\left(\xi_{\alpha} h \otimes \mathfrak{u}_{k}\right)=h \xi_{\alpha} \diamond \xi_{k}, h \in X .
$$

Below we summarize the properties of the operator $\delta$.

Theorem 2.8. If $f$ is an element of $\mathcal{R} L_{2}(\mathbb{F} ; X \otimes \mathcal{U})$ so that $f=\sum_{k \geq 1} f_{k} \otimes \mathfrak{u}_{k}$, with $f_{k}=\sum_{\alpha \in \mathcal{J}} f_{k, \alpha} \xi_{\alpha} \in \mathcal{R} L_{2}(\mathbb{F} ; X)$, then

$$
\delta(f)=\sum_{k \geq 1} f_{k} \diamond \xi_{k}
$$

and

$$
(\delta(f))_{\alpha}=\sum_{k \geq 1} \sqrt{\alpha_{k}} f_{k, \alpha-\varepsilon_{k}} .
$$

Proof. By linearity and (2.15),

$$
\delta(f)=\sum_{k \geq 1} \sum_{\alpha \in \mathcal{J}} \delta\left(\xi_{\alpha} f_{k, \alpha} \otimes \mathfrak{u}_{k}\right)=\sum_{k \geq 1} \sum_{\alpha \in \mathcal{J}} f_{k, \alpha} \xi_{\alpha} \diamond \xi_{k}=\sum_{k \geq 1} f_{k} \diamond \xi_{k},
$$

which is (2.16). On the other hand, by (2.10),

$$
\delta(f)=\sum_{k \geq 1} \sum_{\alpha \in \mathcal{J}} f_{k, \alpha} \sqrt{\alpha_{k}+1} \xi_{\alpha+\varepsilon_{k}}=\sum_{k \geq 1} \sum_{\alpha \in \mathcal{J}} f_{k, \alpha-\varepsilon_{k}} \sqrt{\alpha_{k}} \xi_{\alpha},
$$

and (2.17) follows.

Remark 2.9. It is not difficult to show that the operator $\delta$ can be considered as an extension of the Skorokhod integral to the weighted spaces $\mathcal{R} L_{2}(\mathbb{F} ; X \otimes \mathcal{U})$.

One way to describe a multi-index $\alpha$ with $|\alpha|=n>0$ is by its characteristic set $K_{\alpha}$, that is, an ordered $n$-tuple $K_{\alpha}=\left\{k_{1}, \ldots, k_{n}\right\}$, where $k_{1} \leq k_{2} \leq \ldots \leq k_{n}$ characterize the locations and the values of the non-zero elements of $\alpha$. More precisely, $k_{1}$ is the index of the first non-zero element of $\alpha$, followed by $\max \left(0, \alpha_{k_{1}}-1\right)$ of entries with the same value. The next entry after that is the index of the second non-zero element of $\alpha$, followed by $\max \left(0, \alpha_{k_{2}}-1\right)$ of entries with the same value, and so on. For example, if $n=7$ and $\alpha=(1,0,2,0,0,1,0,3,0, \ldots)$, then the non-zero elements of $\alpha$ are $\alpha_{1}=1, \alpha_{3}=2, \alpha_{6}=1, \alpha_{8}=3$. As a result, $K_{\alpha}=\{1,3,3,6,8,8,8\}$, that is, $k_{1}=1, k_{2}=k_{3}=3, k_{4}=6, k_{5}=k_{6}=k_{7}=8$. 
Using the notion of the characteristic set, we now state the following analog of the well-known result of Itô [10] connecting multiple Wiener integrals and Hermite polynomials.

Proposition 2.10. Let $\alpha \in \mathcal{J}$ be a multi-index with $|\alpha|=n \geq 1$ and characteristic set $K_{\alpha}=\left\{k_{1}, \ldots, k_{n}\right\}$. Then

$$
\xi_{\alpha}=\frac{\xi_{k_{1}} \diamond \xi_{k_{2}} \diamond \cdots \diamond \xi_{k_{n}}}{\sqrt{\alpha !}}
$$

Proof. This follows from (2.3) and (2.13), because by (2.13), for every $i$ and $k$,

$$
H_{k}\left(\xi_{i}\right)=\underbrace{\xi_{i} \diamond \cdots \diamond \xi_{i}}_{k \text { times }} \text {. }
$$

\section{Evolution Equations Driven by White Noise}

3.1. The setting. In this section we study anticipating solutions of stochastic evolution equations driven by Gaussian white noise on a Hilbert space $\mathcal{U}$.

Definition 3.1. The triple $\left(V, H, V^{\prime}\right)$ of Hilbert spaces is called normal if and only if

(1) $V \hookrightarrow H \hookrightarrow V^{\prime}$ and both embeddings $V \hookrightarrow H$ and $H \hookrightarrow V^{\prime}$ are dense and continuous;

(2) The space $V^{\prime}$ is the dual of $V$ relative to the inner product in $H$;

(3) There exists a constant $C>0$ so that $\left|(h, v)_{H}\right| \leq C\|v\|_{V}\|h\|_{V^{\prime}}$ for all $v \in V$ and $h \in H$.

For example, the Sobolev spaces $\left(H_{2}^{\ell+\gamma}\left(\mathbb{R}^{d}\right), H_{2}^{\ell}\left(\mathbb{R}^{d}\right), H_{2}^{\ell-\gamma}\left(\mathbb{R}^{d}\right)\right), \gamma>0, \ell \in \mathbb{R}$, form a normal triple.

Denote by $\left\langle v^{\prime}, v\right\rangle, v^{\prime} \in V^{\prime}, v \in V$, the duality between $V$ and $V^{\prime}$ relative to the inner product in $H$. The properties of the normal triple imply that $\left|\left\langle v^{\prime}, v\right\rangle\right| \leq C\left\|_{v}\right\|_{V}\left\|v^{\prime}\right\|_{V^{\prime}}$, and, if $v^{\prime} \in H$ and $v \in V$, then $\left\langle v^{\prime}, v\right\rangle=\left(v^{\prime}, v\right)_{H}$.

We will also use the following notation:

$$
\mathcal{V}=L_{2}((0, T) ; V), \mathcal{H}=L_{2}((0, T) ; H), \mathcal{V}^{\prime}=L_{2}\left((0, T) ; V^{\prime}\right) .
$$

Given a normal triple $\left(V, H, V^{\prime}\right)$, let $\mathbf{A}: V \rightarrow V^{\prime}$ and $\mathbf{M}: V \rightarrow V^{\prime} \otimes \mathcal{U}$ be bounded linear operators.

Definition 3.2. The solution of the stochastic evolution equation

$$
\dot{u}=\mathbf{A} u+f+\delta(\mathbf{M} u), 0<t \leq T,
$$

with $f \in \mathcal{R} L_{2}\left(\mathbb{F} ; \mathcal{V}^{\prime}\right)$ and $\left.u\right|_{t=0}=u_{0} \in \mathcal{R} L_{2}(\mathbb{F} ; H)$, is a process $u \in \mathcal{R} L_{2}(\mathbb{F} ; \mathcal{V})$ so that, for every $\varphi$ satisfying $\varphi \in \mathcal{R}^{-1} L_{2}(\mathbb{F})$ and $\mathbf{D} \varphi \in \mathcal{R}^{-1} L_{2}(\mathbb{F} ; \mathcal{U})$, the equality

$$
\langle\langle u(t), \varphi\rangle\rangle=\left\langle\left\langle u_{0}, \varphi\right\rangle\right\rangle+\int_{0}^{t}\langle\langle\mathbf{A} u(s)+f(s)+\delta(\mathbf{M} u)(s), \varphi\rangle\rangle d s
$$


holds in $\mathcal{V}^{\prime}$; see (2.5) for the definition of $\langle\langle\cdot, \cdot\rangle$,$\rangle .$

Remark 3.3. (a) The solutions described by Definitions 3.2 and 4.1 belong to the class of "variational solutions", which is quite typical for partial differential equations (see [14, 16, 17, 28], etc.)

(b) Since $\langle\langle u(t), \varphi\rangle\rangle \in \mathcal{V}$ and $\langle\langle u(t), \varphi\rangle\rangle_{t} \in \mathcal{V}^{\prime}$, by the standard embedding theorem (see e.g. [17, Section 1.2.2]) there exists a version of $\langle\langle u(t), \varphi\rangle\rangle \in \mathbf{C}([0, T] ; H)$. Clearly, one could also select a version of $u(t)$ such that $\langle\langle u(t), \varphi\rangle\rangle \in \mathbf{C}([0, T] ; H)$. In the future, we will consider only this version of the solution. By doing this we ensure that formula (3.3) which is understood as an equality in $\mathcal{V}^{\prime}$ yields $\left.u\right|_{t=0}=u_{0} \in \mathcal{R} L_{2}(\mathbb{F} ; H)$.

Remark 3.4. To simplify the notations and the overall presentation, we assume that $\mathbf{A}$ and $\mathbf{M}$ do not depend on time, even though many of the results in this paper can easily be extended to time-dependent operators.

Fix an orthonormal basis $\mathfrak{U}$ in $\mathcal{U}$. Then, for every $v \in V$, there exists a collection $v_{k} \in V^{\prime}, k \geq 1$, so that

$$
\mathbf{M} v=\sum_{k \geq 1} v_{k} \otimes \mathfrak{u}_{k}
$$

We therefore define the operators $\mathbf{M}_{k}: V \rightarrow V^{\prime}$ by setting $\mathbf{M}_{k} v=v_{k}$ and write

$$
\mathbf{M} v=\sum_{k \geq 1}\left(\mathbf{M}_{k} v\right) \otimes \mathfrak{u}_{k}
$$

By (2.16), equation (3.2) becomes

$$
\dot{u}(t)=\mathbf{A} u(t)+f(t)+\mathbf{M} u(t) \diamond \dot{W},
$$

where

$$
\mathbf{M} v \diamond \dot{W}:=\sum_{k \geq 1}\left(\mathbf{M}_{k} v\right) \diamond \xi_{k}
$$

3.2. Equivalence Theorem. In this section we investigate stochastic Fourier representation of equation (3.4).

Recall that every process $u=u(t)$ from $\mathcal{R} L_{2}(\mathbb{F} ; \mathcal{V})$ is represented by a formal series $u(t)=\sum_{\alpha \in \mathcal{J}} u_{\alpha}(t) \xi_{\alpha}$, with $u_{\alpha} \in \mathcal{V}$ and

$$
\sum_{\alpha} r_{\alpha}^{2}\left\|u_{\alpha}\right\|_{\mathcal{V}}^{2}<\infty
$$

Theorem 3.5. Let $u=\sum_{\alpha \in \mathcal{J}} u_{\alpha} \xi_{\alpha}$ be an element of $\mathcal{R} L_{2}(\mathbb{F} ; \mathcal{V})$. The process $u$ is a solution of equation (3.2) if and only if the functions $u_{\alpha}$ have the following properties:

(1) every $u_{\alpha}$ is an element of $\left.\mathbf{C}([0, T] ; H)\right)$

(2) the system of equalities

$$
u_{\alpha}(t)=u_{0, \alpha}+\int_{0}^{t}\left(\mathbf{A} u_{\alpha}(s)+f_{\alpha}(s)+\sum_{k \geq 1} \sqrt{\alpha_{k}} \mathbf{M}_{k} u_{\alpha-\varepsilon_{k}}(s)\right) d s
$$

holds in $V^{\prime}$ for all $t \in[0, T]$ and $\alpha \in \mathcal{J}$. 
Proof. Let $u$ be a solution of $(3.2)$ in $\mathcal{R} L_{2}(\mathbb{F} ; \mathcal{V})$. Taking $\varphi=\xi_{\alpha}$ in (3.3) and using relation (2.17), we obtain equation (3.7). By Remark $\left.3.3 u_{\alpha} \in \mathcal{V} \cap \mathbf{C}([0, T] ; H)\right)$.

Conversely, let $\left\{u_{\alpha}, \alpha \in \mathcal{J}\right\}$ be a collection of functions from $\left.\mathcal{V} \cap \mathbf{C}([0, T] ; H)\right)$ satisfying (3.6) and (3.7). Set $u(t):=\sum_{\alpha \in \mathcal{J}} u_{\alpha}(t) \xi_{\alpha}$. Then, by Theorem 2.8, equation (3.7) yields that, for every $\alpha \in \mathcal{J}$,

$$
\left\langle\left\langle u(t), \xi_{\alpha}\right\rangle\right\rangle=\left\langle\left\langle u_{0}, \xi_{\alpha}\right\rangle\right\rangle+\int_{0}^{t}\left\langle\left\langle\mathbf{A} u(s)+f(s)+\delta(\mathbf{M} u)(s), \xi_{\alpha}\right\rangle\right\rangle d s .
$$

By continuity, we conclude that for any $\varphi \in \mathcal{R}^{-1} L_{2}(\mathbb{F})$ such that $\mathbf{D} \varphi \in \mathcal{R}^{-1} L_{2}(\mathbb{F} ; \mathcal{U})$, equality

$$
\langle\langle u(t), \varphi\rangle\rangle=\left\langle\left\langle u_{0}, \varphi\right\rangle\right\rangle+\int_{0}^{t}\langle\langle\mathbf{A} u(s)+f(s)+\delta(\mathbf{M} u)(s), \varphi\rangle\rangle d s
$$

holds in $\mathcal{V}^{\prime}$. By Remark $3.3\langle\langle u(t), \varphi\rangle\rangle \in \mathbf{C}([0, T] ; H)$.

This simple but very helpful result establishes the equivalence of the "physical" (3.4) and the (stochastic) Fourier (3.7) forms of equation (3.2). System of equations (3.7) is often referred in the literature as the propagator of equation (3.4). Note that the propagator is lower-triangular and can be solved by induction on $|\alpha|$.

3.3. Existence and uniqueness. Below, we will present several results on existence and uniqueness of evolution equations driven by Gaussian white noise.

Before proceeding with general existence-uniqueness problems, we will introduce two simple examples that indicate the limits of the "quality" of solutions of bi-linear SPDEs driven by general Gaussian white noise.

Example 3.6. Consider equation

$$
u(t)=\phi+\int_{0}^{t}(b u(s) \diamond \xi-\lambda u(s)) d s,
$$

where $\phi, \lambda$ are real numbers, $b$ is a complex number, and $\xi$ is a standard Gaussian random variable. In other words $\xi$ is Gaussian white noise on $\mathcal{U}=\mathbb{R}$. With only one Gaussian random variable $\xi$, the set $\mathcal{J}$ becomes $\{0,1,2, \ldots\}$ so that $u(t)=$ $\sum_{n>0} u_{(n)}(t) H_{n}(\xi) / \sqrt{n !}$, where $H_{n}$ is Hermite polynomial of order $n$ (2.4). According to (3.7),

$$
u_{(n)}(t)=\phi I_{(n=0)}-\int_{0}^{t} \lambda u_{(n)}(s) d s+\int_{0}^{t} b \sqrt{n} u_{(n-1)}(s) d s .
$$

It follows that $u_{(0)}(t)=\phi e^{-\lambda t}$ and then, by induction, $u_{(n)}(t)=\phi \frac{(b t)^{n}}{\sqrt{n !}} e^{-\lambda t}$. As a result,

$$
u(t)=e^{-\lambda t}\left(\phi+\sum_{n \geq 1} \frac{(b t)^{n}}{n !} H_{n}(\xi)\right)=\phi e^{-\lambda t+\left(b t \xi-|b|^{2} t^{2} / 2\right)} .
$$

Obviously, the solution of the equation is square integrable on any fixed time interval. However, as the next example indicates, the solutions of SPDEs driven by stationary 
noise are much more intricate then the non-anticipating, or adapted, solutions of SPDEs driven by space-time white noise.

Example 3.7. With $\xi$ as in the previous examples, consider a partial differential equation

$$
u_{t}(t, x)=a u_{x x}(t, x)+\left(\beta u(t, x)+\sigma u_{x}(t, x)\right) \diamond \xi, t>0, x \in \mathbb{R},
$$

with some initial condition $u_{0} \in L_{2}(\mathbb{R})$. By taking the Fourier transform and using the results of Example 3.6 with $\phi=\hat{u}_{0}(y), \lambda=-a y^{2}, b=\beta+\sqrt{-1} y \sigma$, we find

$$
\begin{aligned}
\hat{u}_{t}(t, y) & =-y^{2} a \hat{u}(t)+(\beta+\sqrt{-1} y \sigma) \hat{u}(t, y) \diamond \xi ; \\
\hat{u}(t, y) & =\hat{u}_{0}(y) \exp \left(-t a y^{2}+\left(\sigma^{2} y^{2}-\beta^{2}\right) t^{2} / 2+\sqrt{-1} \beta \sigma y t^{2}+(\sqrt{-1} \sigma y+\beta) t \xi\right) .
\end{aligned}
$$

If $\sigma=0$, i.e. the "diffusion" operator in equation (3.9) is of order zero, then the solution belongs to $L_{2}\left(\mathbb{F} ; L_{2}(\mathbb{R})\right)$ for all $t$. However, if $\sigma>0$, then the solution $u(t, \cdot)$ will, in general, belong to $L_{2}\left(\mathbb{F} ; L_{2}(\mathbb{R})\right)$ only for $t \leq 2 a / \sigma^{2}$. This blow-up in finite time is in sharp contrast with the solution of the equation

$$
u_{t}=a u_{x x}+\sigma u_{x} \diamond \dot{w}
$$

driven by the standard one-dimensional white noise $\dot{w}(t)=\partial_{t} W(t)$, where $W(t)$ is the one-dimensional Brownian motion; a more familiar way of writing (3.10) is in the Itô form

$$
d u=a u_{x x} d t+\sigma u_{x} d W(t)
$$

It is well known (see, for example, [28]) that the solution of (3.11) belongs to $L_{2}\left(\mathbb{F} ; L_{2}(\mathbb{R})\right)$ for every $t>0$ as long as $u_{0} \in L_{2}(\mathbb{R})$ and

$$
a-\sigma^{2} / 2 \geq 0
$$

The existence of a square integrable (global) solution of an Itô's SPDE with square integrable initial condition hinges on the parabolic condition which in the case of equation (3.10) is given by (3.12). Example 3.7 shows that this condition is not in any way sufficient for SPDEs involving a Skorokhod-type integral. The next theorem provides sufficient conditions for the existence and uniqueness of a solution to equation (3.4) in the space $\mathcal{R} L_{2}(\mathbb{F} ; \mathcal{V})$, which appears to be a reasonable extension of the class of square integrable solutions.

Firstly, we introduce an additional assumption on the operator $\mathbf{A}$ that will be used throughout this section:

(A): For every $U_{0} \in H$ and $F \in \mathcal{V}^{\prime}:=L_{2}\left((0, T) ; V^{\prime}\right)$, there exists a function $U \in \mathcal{V}$ that solve the deterministic equation

$$
\partial_{t} U(t)=\mathbf{A} U(t)+F(t), U(0)=U_{0},
$$

and there exists a constant $C=C(\mathbf{A}, T)$ so that

$$
\|U\|_{\mathcal{V}} \leq C(\mathbf{A}, T)\left(\left\|U_{0}\right\|_{H}+\|F\|_{\mathcal{V}^{\prime}}\right)
$$


Remark 3.8. Assumption (A) implies that a solution of equation (3.13) is unique and belongs to $\mathbf{C}((0, T) ; H)$ (cf. Remark 3.3). The assumption also implies that the operator A generates a semi-group $\Phi=\Phi_{t}, t \geq 0$, and, for every $v \in \mathcal{V}$,

$$
\int_{0}^{T}\left\|\int_{0}^{t} \Phi_{t-s} \mathbf{M}_{k} v(s) d s\right\|_{V}^{2} d t \leq C_{k}^{2}\|v\|_{\mathcal{V}}^{2},
$$

with numbers $C_{k}$ independent of $v$.

Remark 3.9. There are various types of assumptions on the operator $\mathbf{A}$ that yield the statement of the assumption (A). In particular, (A) holds if the operator $\mathbf{A}$ is coercive in $\left(V, H, V^{\prime}\right)$ :

$$
\langle\mathbf{A} v, v\rangle+\gamma\|v\|_{V}^{2} \leq C\|v\|_{H}^{2}
$$

for every $v \in V$, where $\gamma>0$ and $C \in \mathbb{R}$ are both independent of $v$.

Theorem 3.10. Assume(A). Consider equation (3.4) in which $u_{0} \in \overline{\mathcal{R}} L_{2}(\mathbb{F} ; H)$, $f \in \overline{\mathcal{R}} L_{2}\left(\mathbb{F} ; \mathcal{V}^{\prime}\right)$ for some operator $\overline{\mathcal{R}}$, and each $\mathbf{M}_{k}$ is a bounded linear operator from $V$ to $V^{\prime}$.

Then there exist an operator $\mathcal{R}$ and a unique solution $u \in \mathcal{R} L_{2}(\mathbb{F} ; \mathcal{V})$ of (3.4).

Proof. By Theorem 3.5, it suffices to prove that the propagator (3.7) has a unique solution $\left(u_{\alpha}(t)\right)_{\alpha \in \mathcal{J}}$ such that for each $\alpha, u_{\alpha} \in \mathcal{V} \cap \mathbf{C}([0, T] ; H)$ and $u:=\sum_{\alpha \in \mathcal{J}} u_{\alpha} \xi_{\alpha} \in$ $\mathcal{R} L_{2}(\mathbb{F} ; \mathcal{V})$.

For $\alpha=(0)$, that is, when $|\alpha|=0$, equation (3.7) reduces to

$$
u_{(0)}(t)=u_{0,(0)}+\int_{0}^{t}\left(\mathbf{A} u_{(0)}+f_{(0)}\right)(s) d s .
$$

By (A), this equation has a unique solution and

$$
\left\|u_{(0)}\right\|_{\mathcal{V}} \leq C(\mathbf{A}, T)\left(\left\|u_{0,(0)}\right\|_{H}+\left\|f_{(0)}\right\|_{\mathcal{V}^{\prime}}\right) .
$$

Using assumption (A), it follows by induction on $|\alpha|$ that, for every $\alpha \in \mathcal{J}$, equation

$$
\partial_{t} u_{\alpha}(t)=\mathbf{A} u_{\alpha}(t)+f_{\alpha}(t)+\sum_{k \geq 1} \sqrt{\alpha_{k}} \mathbf{M}_{k} u_{\alpha-\varepsilon_{k}}(t), u_{\alpha}(0)=u_{0, \alpha}
$$

has a unique solution in $\mathcal{V} \cap \mathbf{C}([0, T] ; H)$. Moreover, by (3.14),

$$
\left\|u_{\alpha}\right\|_{\mathcal{V}} \leq \bar{C}(\mathbf{A}, \mathbf{M}, T)\left(\left\|u_{0, \alpha}\right\|_{H}+\left\|f_{\alpha}\right\|_{\mathcal{V}^{\prime}}+\sum_{k \geq 1} \sqrt{\alpha_{k}}\left\|u_{\alpha-\varepsilon_{k}}\right\|_{\mathcal{V}}\right) .
$$

Since only finitely many of $\alpha_{k}$ are different from 0 , we conclude that $\left\|u_{\alpha}\right\|_{\mathcal{V}}<\infty$ for all $\alpha \in \mathcal{J}$.

Define the operator $\mathcal{R}$ on $L_{2}(\mathbb{F})$ using the weights

$$
r_{\alpha}=\min \left(\bar{r}_{\alpha}, \frac{(2 \mathbb{N})^{-\kappa \alpha}}{1+\left\|u_{\alpha}\right\|_{\mathcal{V}}}\right)
$$

where $\kappa>1 / 2$ (cf. (2.6) $)$. Then $u(t):=\sum_{\alpha \in \mathcal{J}} u_{\alpha}(t) \xi_{\alpha}$ is a solution of (3.4) and, by (2.14), belongs to $\mathcal{R} L_{2}(\mathbb{F} ; \mathcal{V})$. 
While Theorem 3.10 establishes that under very broad assumptions one can find an operator $\mathcal{R}$ such that equation (3.4) has a unique solution in $\mathcal{R} L_{2}(\mathbb{F} ; \mathcal{V})$, the choice of the operator $\mathcal{R}$ is not sufficiently explicit (because of the presence of $\left\|u_{\alpha}\right\|_{\mathcal{V}}$ ) and is not necessarily optimal.

Consider equation (3.4) with non-random $f$ and $u_{0}$. In this situation, it is possible to find more constructive expression for $r_{\alpha}$ and to derive explicit formulas, both for $\mathcal{R} u$ and for each individual $u_{\alpha}$.

Theorem 3.11. If $u_{0}$ and $f$ are non-random, then the following holds:

(1) the coefficient $u_{\alpha}$, corresponding to the multi-index $\alpha$ with $|\alpha|=n \geq 1$ and characteristic set $K_{\alpha}=\left\{k_{1}, \ldots, k_{n}\right\}$, is given by

$$
u_{\alpha}(t)=\frac{1}{\sqrt{\alpha !}} \sum_{\sigma \in \mathcal{P}_{n}} \int_{0}^{t} \int_{0}^{s_{n}} \ldots \int_{0}^{s_{2}} \Phi_{t-s_{n}} \mathbf{M}_{k_{\sigma(n)}} \ldots \Phi_{s_{2}-s_{1}} \mathbf{M}_{k_{\sigma(1)}} u_{(0)}\left(s_{1}\right) d s_{1} \ldots d s_{n}
$$

where

- $\mathcal{P}_{n}$ is the permutation group of the set $(1, \ldots, n)$;

- $\Phi_{t}$ is the semigroup generated by $\mathbf{A}$;

- $u_{(0)}(t)=\Phi_{t} u_{0}+\int_{0}^{t} \Phi_{t-s} f(s) d s$.

(2) the weights $r_{\alpha}$ can be taken in the form

$$
r_{\alpha}=\frac{q^{\alpha}}{\sqrt{|\alpha| !}}, \text { where } q^{\alpha}=\prod_{k=1}^{\infty} q_{k}^{\alpha_{k}}
$$

and the numbers $q_{k}, k \geq 1$, are chosen so that $\sum_{k \geq 1} q_{k}^{2} C_{k}^{2}<1$, with $C_{k}$ from (3.15).

(3) With $q_{k}$ and $r_{\alpha}$ from (3.18), we have

$$
\begin{aligned}
& \sum_{|\alpha|=n} q^{\alpha} u_{\alpha}(t) \xi_{\alpha}=\int_{0}^{t} \int_{0}^{s_{n}} \ldots \int_{0}^{s_{2}} \Phi_{t-s_{n}} \delta\left(\overline{\mathbf{M}} \Phi_{s_{n}-s_{n-1}} \delta\left(\ldots \delta\left(\overline{\mathbf{M}} u_{(0)}\right)\right) \ldots\right) d s_{1} \ldots d s_{n-1} d s_{n}, \\
& \text { where } \overline{\mathbf{M}}=\left(q_{1} \mathbf{M}_{1}, q_{2} \mathbf{M}_{2}, \ldots\right), \text { and } \\
& \mathcal{R} u(t)=u_{(0)}(t) \\
& +\sum_{n=1}^{\infty} \frac{1}{2^{n} \sqrt{n !}} \int_{0}^{t} \int_{0}^{s_{n}} \ldots \int_{0}^{s_{2}} \Phi_{t-s_{n}} \delta\left(\overline{\mathbf{M}} \Phi_{s_{n}-s_{n-1}} \delta\left(\ldots \delta\left(\overline{\mathbf{M}} u_{0}\left(s_{1}\right)\right)\right) \ldots\right) d s_{1} \ldots d s_{n-1} d s_{n} .
\end{aligned}
$$

Proof. If $u_{0}$ and $f$ are deterministic, then equation (3.7) becomes

$$
\begin{aligned}
u_{(0)}(t) & =u_{0}+\int_{0}^{t} \mathbf{A} u_{(0)}(s) d s+\int_{0}^{t} f(s) d s,|\alpha|=0 ; \\
u_{\alpha}(t) & =\int_{0}^{t} \mathbf{A} u_{\alpha}(s) d s+\sum_{k \geq 1} \sqrt{\alpha_{k}} \int_{0}^{t} \mathbf{M}_{k} u_{\alpha-\varepsilon_{k}}(s) d s,|\alpha|>0 .
\end{aligned}
$$


Define $\widetilde{u}_{\alpha}=\sqrt{\alpha !} u_{\alpha}$. Then $\widetilde{u}_{(0)}=u_{(0)}$ and, for $|\alpha|>0$, (3.22) implies

$$
\widetilde{u}_{\alpha}(t)=\int_{0}^{t} \mathbf{A} \widetilde{u}_{\alpha}(s) d s+\sum_{k \geq 1} \int_{0}^{t} \alpha_{k} \mathbf{M}_{k} \widetilde{u}_{\alpha-\varepsilon_{k}}(s) d s
$$

or

$$
\widetilde{u}_{\alpha}(t)=\sum_{k \geq 1} \alpha_{k} \int_{0}^{t} \Phi_{t-s} \mathbf{M}_{k} \tilde{u}_{\alpha-\varepsilon_{k}}(s) d s=\sum_{k \in K_{\alpha}} \int_{0}^{t} \Phi_{t-s} \mathbf{M}_{k} \tilde{u}_{\alpha-\varepsilon_{k}}(s) d s .
$$

By induction on $n$,

$$
\widetilde{u}_{\alpha}(t)=\sum_{\sigma \in \mathcal{P}_{n}} \int_{0}^{t} \int_{0}^{s_{n}} \ldots \int_{0}^{s_{2}} \Phi_{t-s_{n}} \mathbf{M}_{k_{\sigma(n)}} \ldots \Phi_{s_{2}-s_{1}} \mathbf{M}_{k_{\sigma(1)}} u_{(0)} d s_{1} \ldots d s_{n},
$$

and (3.17) follows.

Since (3.20) follows directly from (3.19), it remains to establish (3.19). To this end, define

$$
U_{n}(t)=\sum_{|\alpha|=n} q^{\alpha} u_{\alpha}(t) \xi_{\alpha}, n \geq 0 .
$$

Let us first show that, for each $n \geq 1, U_{n} \in L_{2}(\mathbb{F} ; \mathcal{V})$. Indeed, for $\alpha=(0), u_{\alpha}(0)=u_{0}$, $f_{\alpha}=f$ and

$$
u_{(0)}(t)=\Phi_{t} u_{0}+\int_{0}^{t} \Phi_{t-s} f(s) d s .
$$

By (3.14), we have

$$
\left\|u_{(0)}\right\|_{\mathcal{V}} \leq C(\mathbf{A}, T)\left(\left\|u_{0}\right\|_{H}+\|f\|_{\mathcal{V}^{\prime}}\right) .
$$

When $|\alpha| \geq 1, f_{\alpha}=0$ and the solution of (3.22) is given by

$$
u_{\alpha}(t)=\sum_{k \geq 1} \sqrt{\alpha_{k}} \int_{0}^{t} \Phi_{t-s} \mathbf{M}_{k} u_{\alpha-\varepsilon_{k}}(s) d s .
$$

By (3.17), together with (3.14), (3.23), and (3.15), we have

$$
\left\|u_{\alpha}\right\|_{\mathcal{V}}^{2} \leq C^{2}(\mathbf{A}, T) \frac{(|\alpha| !)^{2}}{\alpha !}\left(\left\|u_{0}\right\|_{H}^{2}+\|f\|_{\mathcal{V}^{\prime}}^{2}\right) \prod_{k \geq 1} C_{k}^{2 \alpha_{k}}
$$

By the multinomial formula,

$$
\left(\sum_{k \geq 1} x_{k}\right)^{n}=\sum_{|\alpha|=n}\left(\frac{n !}{\alpha !} \prod_{k \geq 1} x_{k}^{\alpha_{k}}\right) .
$$

Then

$$
\begin{aligned}
\sum_{|\alpha|=n} q^{2 \alpha}\left\|u_{\alpha}\right\|_{\mathcal{V}}^{2} & \leq C^{2}(\mathbf{A}, T)\left(\left\|u_{0}\right\|_{H}^{2}+\|f\|_{\mathcal{V}^{\prime}}^{2}\right) n ! \sum_{|\alpha|=n}\left(\frac{n !}{\alpha !} \prod_{k \geq 1}\left(C_{k} q_{k}\right)^{2 \alpha_{k}}\right) \\
& =C^{2}(\mathbf{A}, T)\left(\left\|u_{0}\right\|_{H}^{2}+\|f\|_{\mathcal{V}^{\prime}}^{2}\right) n !\left(\sum_{k \geq 1} C_{k}^{2} q_{k}^{2}\right)^{n}<\infty
\end{aligned}
$$


because of the selection of $q_{k}$, and so $U_{n} \in L_{2}(\mathbb{F} ; \mathcal{V})$. Moreover, if the weights $r_{\alpha}$ are defined by (3.18), then

$$
\sum_{\alpha \in \mathcal{J}} r_{\alpha}^{2}\left\|u_{\alpha}\right\|_{\mathcal{V}}^{2}=\sum_{n \geq 0} \sum_{|\alpha|=n} r_{\alpha}^{2}\left\|u_{\alpha}\right\|_{\mathcal{V}}^{2} \leq C^{2}(\mathbf{A}, T)\left(\left\|u_{0}\right\|_{H}^{2}+\|f\|_{\mathcal{V}^{\prime}}^{2}\right) \sum_{n \geq 1}\left(\sum_{k \geq 1} C_{k}^{2} q_{k}^{2}\right)^{n}<\infty
$$

because of the assumption $\sum_{k \geq 1} C_{k}^{2} q_{k}^{2}<1$.

Next, the definition of $U_{n}(t)$ and (3.24) imply that (3.19) is equivalent to

$$
U_{n}(t)=\int_{0}^{t} \Phi_{t-s} \delta\left(\overline{\mathbf{M}} U_{n-1}(s)\right) d s, n \geq 1 .
$$

Accordingly, we will prove (3.27). For $n=1$, we have

$$
U_{1}(s)=\sum_{k \geq 1} q_{k} u_{\varepsilon_{k}}(t) \xi_{k}=\sum_{k \geq 1} \int_{0}^{t} q_{k} \Phi_{t-s} \mathbf{M}_{k} u_{(0)} \xi_{k} d t=\int_{0}^{t} \Phi_{t-s} \delta\left(\overline{\mathbf{M}} U_{0}(s)\right) d s
$$

where the last equality follows from (2.16). More generally, for $n>1$ we have by definition of $U_{n}$ that

From the equation

$$
\left(U_{n}\right)_{\alpha}(t)= \begin{cases}q^{\alpha} u_{\alpha}(t), & \text { if }|\alpha|=n \\ 0, & \text { otherwise }\end{cases}
$$

$$
q^{\alpha} u_{\alpha}(t)=\int_{0}^{t} \mathbf{A} q^{\alpha} u_{\alpha}(s) d s+\sum_{k \geq 1} \int_{0}^{t} q_{k} \sqrt{\alpha_{k}} \mathbf{M}_{k} q^{\alpha-\varepsilon_{k}} u_{\alpha-\varepsilon_{k}}(s) d s
$$

we find

$$
\begin{aligned}
\left(U_{n}(t)\right)_{\alpha} & = \begin{cases}\sum_{k \geq 1} \sqrt{\alpha_{k}} q_{k} \int_{0}^{t} \Phi_{t-s} \mathbf{M}_{k} q^{\alpha-\varepsilon_{k}} u_{\alpha-\varepsilon_{k}}(s) d s, & \text { if }|\alpha|=n, \\
0, & \text { otherwise. }\end{cases} \\
& =\sum_{k \geq 1} \sqrt{\alpha_{k}} \int_{0}^{t} \Phi_{t-s} \overline{\mathbf{M}}_{k}\left(U_{n-1}(s)\right)_{\alpha-\varepsilon_{k}} d s,
\end{aligned}
$$

and then (3.27) follows from (2.17). Theorem 4.5 is proved.

Formula (3.19) is similar to the multiple Wiener integral representation of the solution of a stochastic parabolic equation driven by the Wiener process; see [19, Theorem 3.8].

Example 3.12. Consider the equation

$$
u(t, x)=u_{0}(x)+\int_{0}^{t} u_{x x}(s, x) d s+\sum_{k \geq 1} \int_{0}^{t} \sigma_{k} u_{x x}(s, x) \diamond \xi_{k} d s .
$$

With no loss of generality assume that $\sigma_{k} \neq 0$ for all $k$. Standard properties of the heat kernel imply assumption (A) and inequality (3.15) with $C_{k}=\sigma_{k}^{2}$. Then the conclusions of Theorem 3.11 hold, and we can take $q_{k}^{2}=k^{-2} 4^{-k}\left(1+\sigma_{k}^{2}\right)^{-k}$. Note that Theorem 3.11 covers equation (3.28) with no restrictions on the numbers $\sigma_{k}$. 
In the existing literature on the subject, equations of the type (3.4) are considered only under the assumption

(H): each $\mathbf{M}_{k}$ is a bounded linear operators from $V$ to $H$.

Obviously this assumption rules out equation (3.28) but still covers equation (3.9).

Of course, Theorem 3.11 does not rule out a possibility of a better-behaving solution under additional assumptions on the operators $\mathbf{M}_{k}$. Indeed, it was shown in [18] that if $(\mathrm{H})$ is assumed and the space-only Gaussian noise in equation (3.4) is replaced by the space-time white noise, then a more delicate analysis of equation (3.4) is possible. In particular, the solution can belong to a much smaller Wiener chaos space even if $u_{0}$ and $f$ are not deterministic.

If the operators $\mathbf{M}_{k}$ are bounded in $H$ (see e.g. equation (3.9) with $\sigma=0$ ), then, as the following theorem shows, the solutions can be square integrable (cf. [9]).

Theorem 3.13. Assume that the operator A satisfies

$$
\langle\mathbf{A} v, v\rangle+\kappa\|v\|_{V}^{2} \leq C_{A}\|v\|_{H}^{2}
$$

for every $v \in V$, with $\kappa>0, C_{A} \in \mathbb{R}$ independent of $v$, and assume that each $\mathbf{M}_{k}$ is a bounded operator on $H$ so that $\left\|\mathbf{M}_{k}\right\|_{H \rightarrow H} \leq c_{k}$ and

$$
C_{M}:=\sum_{k \geq 1} c_{k}^{2}<\infty
$$

If $f \in \mathcal{V}^{\prime}$ and $u_{0} \in H$ are non-random, then there exists a unique solution $u$ of (3.4) so that $u(t) \in L_{2}(\mathbb{F} ; H)$ for every $t$ and

$$
\mathbb{E}\|u(t)\|_{H}^{2} \leq C\left(C_{A}, C_{M}, \kappa, t\right)\left(\int_{0}^{t}\|f(s)\|_{V^{\prime}}^{2} d s+\left\|u_{0}\right\|_{H}^{2}\right) .
$$

Proof. Existence and uniqueness of the solution follow from Theorem 3.10 and Remark 3.9, and it remains to establish (3.31).

It follows from (3.7) that

$$
u_{\alpha}=\frac{1}{\sqrt{\alpha !}} \sum_{k \in K_{\alpha}}^{|\alpha|} \int_{0}^{t} \Phi_{t-s} \mathbf{M}_{k} u_{\alpha-\varepsilon_{k}}(s) d s
$$

where $\Phi$ is the semi-group generated by $\mathbf{A}$ and $K_{\alpha}$ is the characteristic set of $\alpha$. Assumption (3.29) implies that $\left\|\Phi_{t}\right\|_{H \rightarrow H} \leq e^{p t}$ for some $p \in \mathbb{R}$. A straightforward calculation using relation (3.32) and induction on $|\alpha|$ shows that

$$
\left\|u_{\alpha}(t)\right\|_{H} \leq e^{p t} \frac{t^{|\alpha|} c^{\alpha}}{\sqrt{\alpha !}}\left\|u_{(0)}\right\|_{H}
$$

where $c^{\alpha}=\prod_{k} c_{k}^{\alpha_{k}}$ and $u_{(0)}(t)=\Phi_{t} u_{0}+\int_{0}^{t} \Phi_{t-s} f(s) d s$. Assumption (3.29) implies that $\left\|u_{(0)}\right\|_{H}^{2} \leq C\left(C_{A}, \kappa, t\right)\left(\int_{0}^{t}\|f(s)\|_{V^{\prime}}^{2} d s+\left\|u_{0}\right\|_{H}^{2}\right)$. To establish (3.31), it remans to observe that

$$
\sum_{\alpha \in \mathcal{J}} \frac{c^{2 \alpha} t^{2|\alpha|}}{\alpha !}=e^{C_{M} t^{2}}
$$

Theorem 3.13 is proved. 
Remark 3.14. Taking $\mathbf{M}_{k} u=c_{k} u$ shows that, in general, bound (3.33) cannot be improved. When condition (3.30) does not hold, a bound similar to (3.31) can be established in a weighted space $\mathcal{R} L_{2}(\mathbb{F} ; H)$, for example with $r_{\alpha}=q^{\alpha}$, where $q_{k}=$ $1 /\left(2^{k}\left(1+c_{k}\right)\right)$. For special operators $\mathbf{M}_{k}$, a more delicate analysis might be possible; see, for example, [9].

If $f$ and $u_{0}$ are not deterministic, then the solution of (3.4) might not satisfy

$$
\mathbb{E}\|u(t)\|_{H}^{2} \leq C\left(C_{A}, C_{M}, \kappa, t\right)\left(\int_{0}^{t} \mathbb{E}\|f(s)\|_{V^{\prime}}^{2} d s+\mathbb{E}\left\|u_{0}\right\|_{H}^{2}\right)
$$

even if all other conditions of Theorem 3.13 are fulfilled. An example can be constructed similar to Example 9.7 in [18]: an interested reader can verify that the solution of the equation $u(t)=u_{0}+\int_{0}^{t} u(s) \diamond \xi d s$, where $\xi$ is a standard Gaussian random variable and $u_{0}=\sum_{n \geq 0} a_{n} \frac{H_{n}(\xi)}{\sqrt{n !}}$, satisfies $\mathbb{E} u^{2}(1) \geq \frac{1}{10} \sum_{n \geq 1} a_{n}^{2} e^{\sqrt{n}}$. For equations with random input, one possibility is to use the spaces $(\mathcal{S})_{-1, q}$; see (2.6). Examples of the corresponding results are Theorems 4.6 and 5.1 below and Theorem 9.8 in [18].

\section{Stationary EQUATIONS}

4.1. Definitions and Analysis. The objective of this section is to study stationary stochastic equation

$$
\mathbf{A} u+\delta(\mathbf{M} u)=f
$$

Definition 4.1. The solution of equation $\sqrt{4.1})$ with $f \in \mathcal{R} L_{2}\left(\mathbb{F} ; V^{\prime}\right)$, is a random element $u \in \mathcal{R} L_{2}(\mathbb{F} ; V)$ so that, for every $\varphi$ satisfying $\varphi \in \mathcal{R}^{-1} L_{2}(\mathbb{F})$ and $\mathbf{D} \varphi \in$ $\mathcal{R}^{-1} L_{2}(\mathbb{F} ; \mathcal{U})$, the equality

$$
\langle\langle\mathbf{A} u, \varphi\rangle\rangle+\langle\langle\delta(\mathbf{M} u), \varphi\rangle\rangle=\langle\langle f, \varphi\rangle\rangle
$$

holds in $V^{\prime}$.

As with evolution equations, we fix an orthonormal basis $\mathfrak{U}$ in $\mathcal{U}$ and use (2.16) to rewrite (4.1) as

$$
\mathbf{A} u+(\mathbf{M} u) \diamond \dot{W}=f
$$

where

$$
\mathbf{M} u \diamond \dot{W}:=\sum_{k \geq 1} \mathbf{M}_{k} u \diamond \xi_{k}
$$

Taking $\varphi=\xi_{\alpha}$ in (4.2) and using relation (2.17) we conclude, as in Theorem 3.5, that $u=\sum_{\alpha \in \mathcal{J}} u_{\alpha} \xi_{\alpha}$ is a solution of equation (4.1) if and only if $u_{\alpha}$ satisfies

$$
\mathbf{A} u_{\alpha}+\sum_{k \geq 1} \sqrt{\alpha_{k}} \mathbf{M}_{k} u_{\alpha-\varepsilon_{k}}=f_{\alpha}
$$

in the normal triple $\left(V, H, V^{\prime}\right)$. This system of equation is lower-triangular and can be solved by induction on $|\alpha|$.

The following example illucidates the limitations on the "quality" of the solution of equation (4.1). 
Example 4.2. Consider equation

$$
u=1+u \diamond \xi
$$

Similar to Example [3.6, we write $u=\sum_{n \geq 0} u_{(n)} H_{n}(\xi) / \sqrt{n !}$, where $H_{n}$ is Hermite polynomial of order $n$ 2.4). Then (4.5) implies $u_{(n)}=I_{(n=0)}+\sqrt{n} u_{(n-1)}$ or $u_{(0)}=1$, $u_{(n)}=\sqrt{n !}, n \geq 1$, or $u=1+\sum_{n \geq 1} H_{n}(\xi)$. Clearly, the series does not converge in $L_{2}(\mathbb{F})$, but does converge in $(\mathcal{S})_{-1, q}$ for every $q<0$ (see (2.6)). As a result, even a simple stationary equation (4.6) can be solved only in weighted spaces.

Theorem 4.3. Consider equation (4.3) in which $f \in \overline{\mathcal{R}} L_{2}\left(\mathbb{F} ; V^{\prime}\right)$ for some $\overline{\mathcal{R}}$.

Assume that the deterministic equation $\mathbf{A} U=F$ is uniquely solvable in the normal triple $\left(V, H, V^{\prime}\right)$, that is, for every $F \in V^{\prime}$, there exists a unique solution $U=\mathbf{A}^{-1} F \in$ $V$ so that $\|U\|_{V} \leq C_{A}\|F\|_{V^{\prime}}$. Assume also that each $\mathbf{M}_{k}$ is a bounded linear operator from $V$ to $V^{\prime}$ so that, for all $v \in V$

$$
\left\|\mathbf{A}^{-1} \mathbf{M}_{k} v\right\|_{V} \leq C_{k}\|v\|_{V}
$$

with $C_{k}$ independent of $v$.

Then there exists an operator $\mathcal{R}$ and a unique solution $u \in \mathcal{R} L_{2}(\mathbb{F} ; V)$ of (3.4).

Proof. The argument is identical to the proof of Theorem 3.10 .

Remark 4.4. The assumption of the theorem about solvability of the deterministic equation holds if the operator $\mathbf{A}$ satisfies $\langle\mathbf{A} v, v\rangle \geq \kappa\|v\|_{V}^{2}$ for every $v \in V$, with $\kappa>0$ independent of $v$.

An analog of Theorem 3.11 exists if $f$ is non-random. With no time variable, we introduce the following notation to write multiple integrals in the time-independent setting:

$$
\delta_{\mathbf{B}}^{(0)}(\eta)=\eta, \delta_{\mathbf{B}}^{(n)}(\eta)=\delta\left(\mathbf{B} \delta_{\mathbf{B}}^{(n-1)}(\eta)\right), \eta \in \mathcal{R} L_{2}(\mathbb{F} ; V),
$$

where $\mathbf{B}$ is a bounded linear operator from $V$ to $V \otimes \mathcal{U}$.

Theorem 4.5. Under the assumptions of Theorem 4.3, if $f$ is non-random, then the following holds:

(1) the coefficient $u_{\alpha}$, corresponding to the multi-index $\alpha$ with $|\alpha|=n \geq 1$ and the characteristic set $K_{\alpha}=\left\{k_{1}, \ldots, k_{n}\right\}$, is given by

$$
u_{\alpha}=\frac{1}{\sqrt{\alpha !}} \sum_{\sigma \in \mathcal{P}_{n}} \mathbf{B}_{k_{\sigma(n)}} \cdots \mathbf{B}_{k_{\sigma(1)}} u_{(0)},
$$

where

- $\mathcal{P}_{n}$ is the permutation group of the set $(1, \ldots, n)$;

- $\mathbf{B}_{k}=-\mathbf{A}^{-1} \mathbf{M}_{k}$;

- $u_{(0)}=\mathbf{A}^{-1} f$.

(2) the operator $\mathcal{R}$ can be defined by the weights $r_{\alpha}$ in the form

$$
r_{\alpha}=\frac{q^{\alpha}}{\sqrt{|\alpha| !}}, \text { where } q^{\alpha}=\prod_{k=1}^{\infty} q_{k}^{\alpha_{k}}
$$


where the numbers $q_{k}, k \geq 1$ are chosen so that $\sum_{k \geq 1} q_{k}^{2} C_{k}^{2}<1$, and $C_{k}$ are defined in 4.7.

(3) With $r_{\alpha}$ and $q_{k}$ defined by (4.9),

$$
\sum_{|\alpha|=n} q^{\alpha} u_{\alpha} \xi_{\alpha}=\delta_{\overline{\mathbf{B}}}^{(n)}\left(\mathbf{A}^{-1} f\right),
$$

where $\overline{\mathbf{B}}=-\left(q_{1} \mathbf{A}^{-1} \mathbf{M}_{1}, q_{2} \mathbf{A}^{-1} \mathbf{M}_{2}, \ldots\right)$, and

$$
\mathcal{R} u=\mathbf{A}^{-1} f+\sum_{n \geq 1} \frac{1}{\sqrt{n !}} \delta_{\overline{\mathbf{B}}}^{(n)}\left(\mathbf{A}^{-1} f\right),
$$

Proof. While the proofs of Theorems 3.11 and 4.5 are similar, the complete absence of time makes equation (4.3) different from either (3.4) or anything considered in [19]. Accordingly, we present a complete proof.

Define $\widetilde{u}_{\alpha}=\sqrt{\alpha !} u_{\alpha}$. If $f$ is deterministic, then $\widetilde{u}_{(0)}=\mathbf{A}^{-1} f$ and, for $|\alpha| \geq 1$,

or

$$
\mathbf{A} \widetilde{u}_{\alpha}+\sum_{k \geq 1} \alpha_{k} \mathbf{M}_{k} \widetilde{u}_{\alpha-\varepsilon_{k}}=0
$$

$$
\widetilde{u}_{\alpha}=\sum_{k \geq 1} \alpha_{k} \mathbf{B}_{k} \widetilde{u}_{\alpha-\varepsilon_{k}}=\sum_{k \in K_{\alpha}} \mathbf{B}_{k} \widetilde{u}_{\alpha-\varepsilon_{k}}
$$

where $K_{\alpha}=\left\{k_{1}, \ldots, k_{n}\right\}$ is the characteristic set of $\alpha$ and $n=|\alpha|$. By induction on $n$,

$$
\widetilde{u}_{\alpha}=\sum_{\sigma \in \mathcal{P}_{n}} \mathbf{B}_{k_{\sigma(n)}} \cdots \mathbf{B}_{k_{\sigma(1)}} u_{(0)}
$$

and (4.8) follows.

Next, define

$$
U_{n}=\sum_{|\alpha|=n} q^{\alpha} u_{\alpha} \xi_{\alpha}, n \geq 0
$$

Let us first show that, for each $n \geq 1, U_{n} \in L_{2}(\mathbb{F} ; V)$. By (4.8) we have

$$
\left\|u_{\alpha}\right\|_{V}^{2} \leq C_{A}^{2} \frac{(|\alpha| !)^{2}}{\alpha !}\|f\|_{V^{\prime}}^{2} \prod_{k \geq 1} C_{k}^{\alpha_{k}} .
$$

By (3.26),

$$
\begin{aligned}
\sum_{|\alpha|=n} q^{2 \alpha}\left\|u_{\alpha}\right\|_{V}^{2} & \leq C_{A}^{2}\|f\|_{V^{\prime}}^{2} n ! \sum_{|\alpha|=n}\left(\frac{n !}{\alpha !} \prod_{k \geq 1}\left(C_{k} q_{k}\right)^{2 \alpha_{k}}\right) \\
& =C_{A}^{2}\|f\|_{V^{\prime}}^{2} n !\left(\sum_{k \geq 1} C_{k}^{2} q_{k}^{2}\right)^{n}<\infty,
\end{aligned}
$$

because of the selection of $q_{k}$, and so $U_{n} \in L_{2}(\mathbb{F} ; V)$. If the weights $r_{\alpha}$ are defined by (4.9), then

$$
\sum_{\alpha \in \mathcal{J}} r_{\alpha}^{2}\|u\|_{V}^{2}=\sum_{n \geq 0} \sum_{|\alpha|=n} r_{\alpha}^{2}\|u\|_{V}^{2} \leq C_{A}^{2}\|f\|_{V^{\prime}}^{2} \sum_{n \geq 0}\left(\sum_{k \geq 1} C_{k}^{2} q_{k}^{2}\right)^{n}<\infty
$$


because of the assumption $\sum_{k \geq 1} C_{k}^{2} q_{k}^{2}<1$.

Since (4.11) follows directly from (4.10), it remains to establish (4.10), that is,

$$
U_{n}=\delta_{\overline{\mathbf{B}}}\left(U_{n-1}\right), n \geq 1
$$

For $n=1$ we have

$$
U_{1}=\sum_{k \geq 1} q_{k} u_{\varepsilon_{k}} \xi_{k}=\sum_{k \geq 1} \overline{\mathbf{B}}_{k} u_{(0)} \xi_{k}=\delta_{\overline{\mathbf{B}}}\left(U_{0}\right)
$$

where the last equality follows from (2.16). More generally, for $n>1$ we have by definition of $U_{n}$ that

From the equation

$$
\left(U_{n}\right)_{\alpha}= \begin{cases}q^{\alpha} u_{\alpha}, & \text { if }|\alpha|=n \\ 0, & \text { otherwise }\end{cases}
$$

$$
q^{\alpha} \mathbf{A} u_{\alpha}+\sum_{k \geq 1} q_{k} \sqrt{\alpha_{k}} \mathbf{M}_{k} q^{\alpha-\varepsilon_{k}} u_{\alpha-\varepsilon_{k}}=0
$$

we find

$$
\begin{aligned}
\left(U_{n}\right)_{\alpha} & = \begin{cases}\sum_{k \geq 1} \sqrt{\alpha_{k}} q_{k} \mathbf{B}_{k} q^{\alpha-\varepsilon_{k}} u_{\alpha-\varepsilon_{k}}, & \text { if }|\alpha|=n \\
0, & \text { otherwise. }\end{cases} \\
& =\sum_{k \geq 1} \sqrt{\alpha_{k}} \overline{\mathbf{B}}_{k}\left(U_{n-1}\right)_{\alpha-\varepsilon_{k}},
\end{aligned}
$$

and then (4.13) follows from (2.17). Theorem 4.5 is proved.

Here is another result about solvability of (4.3), this time with random $f$. We use the space $(\mathcal{S})_{\rho, q}$, defined by the weights $(2.6)$.

Theorem 4.6. In addition to the assumptions of Theorem 4.3, let $C_{A} \leq 1$ and $C_{k} \leq 1$ for all $k$. If $f \in(\mathcal{S})_{-1,-\ell}\left(V^{\prime}\right)$ for some $\ell>1$, then there exists a unique solution $u \in(\mathcal{S})_{-1,-\ell-4}(V)$ of (4.3) and

$$
\|u\|_{(\mathcal{S})_{-1,-\ell-4}(V)} \leq C(\ell)\|f\|_{(\mathcal{S})_{-1,-\ell}\left(V^{\prime}\right)} .
$$

Proof. Denote by $u(g ; \gamma), \gamma \in \mathcal{J}, g \in V^{\prime}$, the solution of (4.3) with $f_{\alpha}=g I_{(\alpha=\gamma)}$, and define $\bar{u}_{\alpha}=(\alpha !)^{-1 / 2} u_{\alpha}$. Clearly, $u_{\alpha}(g, \gamma)=0$ if $|\alpha|<|\gamma|$ and so

$$
\sum_{\alpha \in \mathcal{J}}\left\|u_{\alpha}\left(f_{\gamma} ; \gamma\right)\right\|_{V}^{2} r_{\alpha}^{2}=\sum_{\alpha \in \mathcal{J}}\left\|u_{\alpha+\gamma}\left(f_{\gamma} ; \gamma\right)\right\|_{V}^{2} r_{\alpha+\gamma}^{2} .
$$

It follows from (4.5) that

$$
\bar{u}_{\alpha+\gamma}\left(f_{\gamma} ; \gamma\right)=\bar{u}_{\alpha}\left(f_{\gamma}(\gamma !)^{-1 / 2} ;(0)\right)
$$

Now we use (4.12) to conclude that

$$
\left\|\bar{u}_{\alpha+\gamma}\left(f_{\gamma} ; \gamma\right)\right\|_{V} \leq \frac{|\alpha| !}{\sqrt{\alpha ! \gamma !}}\|f\|_{V^{\prime}}
$$


Coming back to (4.15) with $r_{\alpha}^{2}=(\alpha !)^{-1}(2 \mathbb{N})^{(-\ell-4) \alpha}$ and using inequality (2.2) we find:

$$
\left\|u\left(f_{\gamma} ; \gamma\right)\right\|_{(\mathcal{S})_{-1,-\ell-4}(V)} \leq C(\ell)(2 \mathbb{N})^{-2 \gamma} \frac{\left\|f_{\gamma}\right\|_{V^{\prime}}}{(2 \mathbb{N})^{(\ell / 2) \gamma} \sqrt{\gamma !}},
$$

where

$$
C(\ell)=\left(\sum_{\alpha \in \mathcal{J}}\left(\frac{|\alpha| !}{\alpha !}\right)^{2}(2 \mathbb{N})^{(-\ell-4) \alpha}\right)^{1 / 2}
$$

(2.14) and (2.2) imply $C(\ell)<\infty$. Then (4.14) follows by the triangle inequality after summing over all $\gamma$ and using the Cauchy-Schwartz inequality.

Remark 4.7. Example [4.2, in which $f \in(\mathcal{S})_{0,0}$ and $u \in(\mathcal{S})_{-1, q}, q<0$, shows that, while the results of Theorem 4.6 are not sharp, a bound of the type $\|u\|_{(\mathcal{S})_{\rho, q}(V)} \leq$ $C\|f\|_{(\mathcal{S})_{\rho, \ell}\left(V^{\prime}\right)}$ is, in general, impossible if $\rho>-1$ or $q \geq \ell$.

4.2. Convergence to Stationary Solution. Let $\left(V, H, V^{\prime}\right)$ be a normal triple of Hilbert spaces. Consider equation

$$
\dot{u}(t)=(\mathbf{A} u(t)+f(t))+\mathbf{M}_{k} u(t) \diamond \xi_{k},
$$

where the operators $\mathbf{A}$ and $\mathbf{M}_{k}$ do not depend on time, and assume that there exists an $f^{*} \in \mathcal{R} L_{2}(\mathbb{F} ; H)$ such that $\lim _{t \rightarrow \infty}\left\|f(t)-f^{*}\right\|_{\mathcal{R} L_{2}(\mathbb{F} ; H)}=0$. The objective of this section is to study convergence, as $t \rightarrow+\infty$, of the solution of (4.18) to the solution $u^{*}$ of the stationary equation

$$
-\mathbf{A} u^{*}=f^{*}+\mathbf{M}_{k} u^{*} \diamond \xi_{k} .
$$

Theorem 4.8. Assume that

(C1) Each $\mathbf{M}_{k}$ is a bounded linear operator from $H$ to $H$, and $\mathbf{A}$ is a bounded linear operator from $V$ to $V^{\prime}$ with the property

$$
\langle\mathbf{A} v, v\rangle+\kappa\|v\|_{V}^{2} \leq-c\|v\|_{H}^{2}
$$

for every $v \in V$, with $\kappa>0$ and $c>0$ both independent of $v$.

(C2) $f \in \overline{\mathcal{R}} L_{2}(\mathbb{F} ; \mathcal{H})$ and there exists an $f^{*} \in \overline{\mathcal{R}} L_{2}(\mathbb{F} ; H)$ such that $\lim _{t \rightarrow+\infty}\left\|f(t)-f^{*}\right\|_{\overline{\mathcal{R}} L_{2}(\mathbb{F} ; H)}$.

Then, for every $u_{0} \in \overline{\mathcal{R}} L_{2}(\mathbb{F} ; H)$, there exists an operator $\mathcal{R}$ so that

(1) There exists a unique solution $u \in \mathcal{R} L_{2}(\mathbb{F} ; \mathcal{V})$ of (4.18),

(2) There exists a unique solution $u^{*} \in \mathcal{R} L_{2}(\mathbb{F} ; V)$ of (4.19), and

(3) The following convergence holds:

$$
\lim _{t \rightarrow+\infty}\left\|u(t)-u^{*}\right\|_{\mathcal{R} L_{2}(\mathbb{F} ; H)}=0 .
$$

Proof (1) Existence and uniqueness of the solution of (4.18) follow from Theorem 3.10 and Remark 3.9.

(2) Existence and uniqueness of the solution of (4.19) follow from Theorem 4.3 and Remark 4.4.

(3) The proof of (4.21) is based on the following result. 
Lemma 4.9. Assume that the operator A satisfies (4.20) and $F=F(t)$ is a deterministic function such that $\lim _{t \rightarrow+\infty}\|F(t)\|_{H}=0$. Then, for every $U_{0} \in H$, the solution $U=U(t)$ of the equation $U(t)=U_{0}+\int_{0}^{t} \mathbf{A} U(s) d s+\int_{0}^{t} F(s) d s$ satisfies $\lim _{t \rightarrow+\infty}\|U(t)\|_{H}=0$.

Proof. If $\Phi=\Phi_{t}$ is the semi-group generated by the operator $\mathbf{A}$ (which exists because of (4.20) ), then

$$
U(t)=\Phi_{t} U_{0}+\int_{0}^{t} \Phi_{t-s} F(s) d s .
$$

Condition (4.20) implies $\left\|\Phi_{t} U_{0}\right\|_{H} \leq e^{-c t}\left\|U_{0}\right\|_{H}$, and then

$$
\|U(t)\|_{H} \leq e^{-c t}\left\|U_{0}\right\|_{H}+\int_{0}^{t} e^{-c(t-s)}\|F(s)\|_{H} d s .
$$

The convergence of $\|U(t)\|_{H}$ to zero now follows from the Toeplitz lemma (see Lemma A.2 in Appendix). Lemma 4.9 is proved.

To complete the proof of Theorem 4.8, we define $v_{\alpha}(t)=u_{\alpha}(t)-u_{\alpha}^{*}$ and note that

$$
\dot{v}_{\alpha}(t)=\mathbf{A} v_{\alpha}(t)+\left(f_{\alpha}(t)-f_{\alpha}^{*}\right)+\sum_{k} \sqrt{\alpha_{k}} \mathbf{M}_{k} v_{\alpha-\varepsilon_{k}}
$$

By Theorem 4.3, $u_{\alpha}^{*} \in V$ and so $v_{\alpha}(0) \in H$ for every $\alpha \in \mathcal{J}$. By Lemma 4.9, $\lim _{t \rightarrow+\infty}\left\|v_{(0)}(t)\right\|_{H}=0$. Using induction on $|\alpha|$ and the inequality $\left\|\mathbf{M}_{k} v_{\alpha-\varepsilon_{k}}(t)\right\|_{H} \leq$ $c_{k}\left\|v_{\alpha-\varepsilon_{k}}(t)\right\|_{H}$, we conclude that $\lim _{t \rightarrow+\infty}\left\|v_{\alpha}(t)\right\|_{H}=0$ for every $\alpha \in \mathcal{J}$. Since $v_{\alpha} \in \mathbf{C}((0, T) ; H)$ for every $T$, it follows that $\sup _{t \geq 0}\left\|v_{\alpha}(t)\right\|_{H}<\infty$. Define the operator $\mathcal{R}$ on $L_{2}(\mathbb{F})$ so that $\mathcal{R} \xi_{\alpha}=r_{\alpha} \xi_{\alpha}$, where

$$
r_{\alpha}=\frac{(2 \mathbb{N})^{-\alpha}}{1+\sup _{t \geq 0}\left\|v_{\alpha}(t)\right\|_{H}} .
$$

Then (4.21) follows by the dominated convergence theorem.

Theorem 4.8 is proved.

\section{Bilinear PARABOliC AND ELliPtic SPDES}

Let $G$ be a smooth bounded domain in $\mathbb{R}^{d}$ and $\left\{h_{k}, k \geq 1\right\}$, an orthonormal basis in $L_{2}(G)$. We assume that

$$
\sup _{x \in G}\left|h_{k}(x)\right| \leq c_{k}, \quad k \geq 1 .
$$

A space white noise on $L_{2}(G)$ is a formal series

$$
\dot{W}(x)=\sum_{k \geq 1} h_{k}(x) \xi_{k}
$$

where $\xi_{k}, k \geq 1$, are independent standard Gaussian random variables. 
5.1. Dirichlet Problem for parabolic SPDE of the Second Order. Consider the following equation:

$$
\begin{aligned}
u_{t}(t, x) & =a_{i j}(x) D_{i} D_{j} u(t, x)+b_{i}(x) D_{i} u(t, x)+c(x) u(t, x)+f(t, x) \\
& +\left(\sigma_{i}(x) D_{i} u(t, x)+\nu(x) u(t, x)+g(t, x)\right) \diamond \dot{W}(x), 0<t \leq T, x \in G,
\end{aligned}
$$

with zero boundary conditions and some initial condition $u(0, x)=u_{0}(x)$; the functions $a_{i j}, b_{i}, c, f, \sigma_{i}, \nu, g$, and $u_{0}$ are non-random. In (5.3) and in similar expressions below we assume summation over the repeated indices. Let $\left(V, H, V^{\prime}\right)$ be the normal triple with $V=\stackrel{\circ}{H_{2}^{1}}(G), H=L_{2}(G), V^{\prime}=H_{2}^{-1}(G)$. In view of (5.2), equation (5.3) is a particular case of equation (3.4) so that

$$
\mathbf{A} u=a_{i j}(x) D_{i} D_{j} u+b_{i}(x) D_{i} u+c(x) u, \mathbf{M}_{k} u=\left(\sigma_{i}(x) D_{i} u+\nu(x) u\right) h_{k}(x),
$$

and $f(t, x)+g(t, x) \diamond \dot{W}(x)$ is the free term.

We make the following assumptions about the coefficients:

D1 The functions $a_{i j}$ are Lipschitz continuous in the closure $\bar{G}$ of $G$, and the functions $b_{i}, c, \sigma_{i}, \nu$ are bounded and measurable in $\bar{G}$.

D2 There exist positive numbers $A_{1}, A_{2}$ so that $A_{1}|y|^{2} \leq a_{i j}(x) y_{i} y_{j} \leq A_{2}|y|^{2}$ for all $x \in \bar{G}$ and $y \in \mathbb{R}^{d}$.

Given a $T>0$, recall the notation $\mathcal{V}=L_{2}((0, T) ; V)$ and similarly for $\mathcal{H}$ and $\mathcal{V}^{\prime}$ (see (3.1) $)$.

Theorem 5.1. Under the assumptions $\boldsymbol{D} 1$ and $\boldsymbol{D} 2$, if $f \in \mathcal{V}^{\prime}, g \in \mathcal{H}, u_{0} \in H$, then there exists an $\ell>1$ and a number $C>0$, both independent of $u_{0}, f, g$, so that $u \in \mathcal{R} L_{2}(\mathbb{F} ; \mathcal{V})$ and

$$
\|u\|_{\mathcal{R} L_{2}(\mathbb{F} ; \mathcal{V})} \leq C \cdot\left(\left\|u_{0}\right\|_{H}+\|f\|_{\mathcal{V}^{\prime}}+\|g\|_{\mathcal{H}}\right)
$$

where the operator $\mathcal{R}$ is defined by the weights

$$
r_{\alpha}^{2}=c^{-2 \alpha}(|\alpha| !)^{-1}(2 \mathbb{N})^{-2 \ell \alpha}
$$

and $c^{\alpha}=\prod_{k} c_{k}^{\alpha_{k}}$, with $c_{k}$ from (5.1); the number $\ell$ in general depends on $T$.

Proof. We derive the result from Theorem 3.11. Consider the deterministic equation $\dot{U}(t)=\mathbf{A} U(t)+F$. Assumptions D1 and D2 imply that there exists a unique solution of this equation in the normal triple $\left(V, H, V^{\prime}\right)$, and the solution satisfies

$$
\sup _{0<t<T}\|U(t)\|_{H}+\|U\|_{\mathcal{V}} \leq C \cdot\left(\|U(0)\|_{H}+\|F\|_{\mathcal{V}^{\prime}}\right)
$$

where the number $C$ depends on $T$ and the operator A. Moreover, (5.1) implies that (3.15) holds with $C_{k}=C_{0} c_{k}$ for some positive number $C_{0}$ independent of $k$, but possibly depending on $T$.

To proceed, let us assume first that $g=0$. Then the statement of the theorem follows directly from Theorem 3.11 if we take in (3.18) $q_{k}=c_{k}{ }^{-1}(2 k)^{-\ell}$ with sufficiently large $\ell$. 
It now remains to consider the case $g \neq 0$ and $f=u_{0}=0$. Even though $g$ is nonrandom, $g \xi_{k}$ is, and therefore a direct application of Theorem 3.11 is not possible. Instead, let us look more closely at the corresponding equations for $u_{\alpha}$. For $\alpha=(0)$,

$$
u_{(0)}(t)=\int_{0}^{t} \mathbf{A} u_{(0)}(s) d s,
$$

which implies $u_{(0)}(t)=0$ for all $t$. For $\alpha=\varepsilon_{k}$,

$$
u_{\varepsilon_{k}}(t)=\int_{0}^{t} \mathbf{A} u_{\varepsilon_{k}}(s) d s+h_{k} \int_{0}^{t} g(s) d s
$$

or

$$
u_{\varepsilon_{k}}(t)=\int_{0}^{t} \Phi_{t-s} h_{k} g(s) d s
$$

so that

$$
\left\|u_{\varepsilon_{k}}\right\|_{\mathcal{V}} \leq C_{0} c_{k}\|g\|_{\mathcal{H}}
$$

If $|\alpha|>1$, then

$$
u_{\alpha}(t)=\int_{0}^{t} \mathbf{A} u_{\alpha}(s) d s+\sum_{k \geq 1} \sqrt{\alpha_{k}} \mathbf{M}_{k} u_{\alpha-\varepsilon_{k}}
$$

which is the same as (3.22). In particular, if $|\alpha|=2$ and $\{i, j\}$ is the characteristic set of $\alpha$, then

$$
u_{\alpha}(t)=\frac{1}{\sqrt{\alpha !}} \int_{0}^{t} \Phi_{t-s}\left(\mathbf{M}_{i} u_{\varepsilon_{j}}(s)+\mathbf{M}_{j} u_{\varepsilon_{i}}(s)\right) d s .
$$

More generally, by analogy with (3.25), if $|\alpha|=n>2$ and $\left\{k_{1}, \ldots, k_{n}\right\}$ is the characteristic set of $\alpha$, then

$$
u_{\alpha}(t)=\frac{1}{\sqrt{\alpha !}} \sum_{\sigma \in \mathcal{P}_{n}} \int_{0}^{t} \int_{0}^{s_{n}} \ldots \int_{0}^{s_{3}} \Phi_{t-s_{n}} \mathbf{M}_{k_{\sigma(n)}} \cdots \Phi_{s_{3}-s_{2}} \mathbf{M}_{k_{\sigma(2)}} u_{\varepsilon_{\sigma(1)}}\left(s_{2}\right) d s_{2} \ldots d s_{n} .
$$

By the triangle inequality and (5.8),

$$
\left\|u_{\alpha}\right\|_{\mathcal{V}} \leq \frac{|\alpha| ! C_{0}^{|\alpha|} c^{\alpha}}{\sqrt{\alpha !}}\|g\|_{\mathcal{H}}
$$

and then (5.5) follows from (2.2) if $\ell$ is sufficiently large.

This completes the proof of Theorem 5.1 .

Theorem 5.2. In addition to $\mathbf{D} 1$ and $\mathbf{D} 2$, assume that

(1) $\sigma_{i}=0$ for all $i$

(2) the operator $\mathbf{A}$ in $G$ with zero boundary conditions satisfies (4.20).

If there exist functions $f^{*}$ and $g^{*}$ from $H$ so that

$$
\lim _{t \rightarrow+\infty}\left(\left\|f(t)-f^{*}\right\|_{H}+\left\|g(t)-g^{*}\right\|_{H}\right)=0,
$$

then the solution $u$ of equation (5.3) satisfies

$$
\lim _{t \rightarrow+\infty}\left\|u(t)-u^{*}\right\|_{\mathcal{R} L_{2}(\mathbb{F} ; H)}=0,
$$


where the operator $\mathcal{R}$ is defined by the weights (5.6) and $u^{*}$ is the solution of the stationary equation

$$
\begin{aligned}
a_{i j}(x) D_{i} D_{j} u^{*}(x) & +b_{i}(x) D_{i} u^{*}(x)+c(x) u^{*}(x)+f^{*}(x) \\
& +\left(\nu(x) u^{*}(x)+g^{*}(x)\right) \diamond \dot{W}(x)=0, x \in G ;\left.u\right|_{\partial G}=0 .
\end{aligned}
$$

Proof. This follows from Theorem 4.8.

Remark 5.3. The operator A satisfies (4.20) if, for example, each $a_{i j}$ is twice continuously differentiable in $\bar{G}$, each $b_{i}$ continuously differentiable in $\bar{G}$, and

$$
\inf _{x \in \bar{G}} c(x)-\sup _{x \in \bar{G}}\left(\left|D_{i} D_{j} a_{i j}(x)\right|+\left|D_{i} b_{i}(x)\right|\right) \geq \varepsilon>0 ;
$$

this is verified directly using integration by parts.

5.2. Elliptic SPDEs of the full second order. Consider the following Dirichlet problem:

$$
\begin{gathered}
-D_{i}\left(a_{i j}(x) D_{j} u(x)\right)+ \\
D_{i}\left(\sigma_{i j}(x) D_{j}(u(x))\right) \diamond \dot{W}(x)=f(x), x \in G, \\
u_{\mid \partial G}=0,
\end{gathered}
$$

where $\dot{W}$ is the space white noise (5.2). Assume that the functions $a_{i j}, \sigma_{i j}, f$, and $g$ are non-random. Recall that according to our summation convention, in (5.13) and in similar expressions below we assume summation over the repeated indices.

We make the following assumptions:

E1: The functions $a_{i j}=a_{i j}(x)$ and $\sigma_{i j}=\sigma_{i j}(x)$ are measurable and bounded in the closure $\bar{G}$ of $G$.

E2: There exist positive numbers $A_{1}, A_{2}$ so that $A_{1}|y|^{2} \leq a_{i j}(x) y_{i} y_{j} \leq A_{2}|y|^{2}$ for all $x \in \bar{G}$ and $y \in \mathbb{R}^{d}$.

E3: The functions $h_{k}$ in (5.2) are bounded and Lipschitz continuous.

Clearly, equation (5.13) is a particular case of equation (4.3) with

$$
\mathbf{A} u(x):=-D_{i}\left(a_{i j}(x) D_{j} u(x)\right)
$$

and

$$
\mathbf{M}_{k} u(x):=h_{k}(x) D_{i}\left(\sigma_{i j}(x) D_{j} u(x)\right) .
$$

Assumptions E1 and E3 imply that each $\mathbf{M}_{k}$ is a bounded linear operator from $\mathrm{H}_{2}^{1}(G)$ to $H_{2}^{-1}(G)$. Moreover, it is a standard fact that under the assumptions E1 and E2 the operator $\mathbf{A}$ is an isomorphism from $V$ onto $V^{\prime}$ (see e.g. [17]). Therefore, for every $k$ there exists a positive number $C_{k}$ such that

$$
\left\|\mathbf{A}^{-1} M_{k} v\right\|_{V} \leq C_{k}\|v\|_{V}, v \in V \text {. }
$$

Theorem 5.4. Under the assumptions $\boldsymbol{E} 1$ and $\boldsymbol{E} 2$, if $f \in H_{2}^{-1}(G)$, then there exists a unique solution of the Dirichlet problem (5.13) $u \in \mathcal{R} L_{2}\left(\mathbb{F} ; H_{2}^{1}(G)\right)$ such that

$$
\|u\|_{\mathcal{R} L_{2}\left(\mathbb{F} ; H_{2}^{1}(G)\right)} \leq C \cdot\|f\|_{H_{2}^{-1}(G)} .
$$


The weights $r_{\alpha}$ can be taken in the form

$$
r_{\alpha}=\frac{q^{\alpha}}{\sqrt{|\alpha| !}}, \text { where } q^{\alpha}=\prod_{k=1}^{\infty} q_{k}^{\alpha_{k}},
$$

and the numbers $q_{k}, k \geq 1$ are chosen so that $\sum_{k \geq 1} C_{k}^{2} q_{k}^{2}<1$, with $C_{k}$ from (5.16).

Proof. This follows from Theorem 4.5.

Remark 5.5. With an appropriate change of the boundary conditions, and with extra regularity of the basis functions $h_{k}$, the results of Theorem 5.4 can be extended to stochastic elliptic equations of order $2 \mathrm{~m}$. The corresponding operators are

$$
\mathbf{A} u=(-1)^{m} D_{i_{1}} \cdots D_{i_{m}}\left(a_{i_{1} \ldots i_{m} j_{1} \ldots j_{m}}(x) D_{j_{1}} \cdots D_{j_{m}} u(x)\right)
$$

and

$$
\mathbf{M}_{k} u=h_{k}(x) D_{i_{1}} \cdots D_{i_{m}}\left(\sigma_{i_{1} \ldots i_{m} j_{1} \ldots j_{m}}(x) D_{j_{1}} \cdots D_{j_{m}} u(x)\right) .
$$

Since $G$ is a smooth bounded domain, regularity of $h_{k}$ is not a problem: we can take $h_{k}$ as the eigenfunctions of the Dirichlet Laplacian in $G$.

\section{REFERENCES}

[1] R. H. Cameron and W. T. Martin, The orthogonal development of nonlinear functionals in a series of Fourier-Hermite functions, Ann. Math. 48 (1947), no. 2, 385-392.

[2] R. E. Carmona, S. A. Molchanov, Parabolic Anderson Problem and Intermittency, Memoirs of the AMS, 108 (1994) no. 518.

[3] R. E. Carmona and B. Rozovskii (Editors) Stochastic Partial Differential Equations: Six Perspectives, Math. Surveys Monogr. 64, AMS, Providence, RI, 1999

[4] DaPrato, G. and Zabczyk, J., Stochastic Equations in Infinite Dimensions, Cambridge University Press, Cambridge, 1992.

[5] Donati-Martin, C. Quazi-linear elliptic stochastic partial differential equations: Markov property, Stochastics Stochastics Rep., 41 (1992), 219-240.

[6] K. Gawȩdzki and M. Vergassola, Phase Transition in the Passive Scalar Advection, Physica D $138(2000), 63-90$.

[7] K. Gawedzki and A. Kupiainen, Universality in Turbulence: an Exactly Solvable Model, Lowdimensional Models in Statistical Physics and Quantum Field Theory, Springer, Berlin, 1996, pp. $71-105$.

[8] T. How, W. Luo, B. Rozovskii, H.-M. Zhou, Wiener chaos expansions and numerical solutions of randomly forced equations of fluid mechanics, J. Comput. Phys. 216 (2006), no. 2, 687-706.

[9] Y. Hu, Chaos expansion of heat equations with white noise potentials, Potential Anal. 16 (2002), no. $1,45-66$.

[10] K. Ito, Multiple Wiener integral, J. Math. Soc. Japan 3 (1951), 157-169.

[11] Yu. G. Kondratiev and Yu. S. Samoylenko, The spaces of trial and generalized functions of infinite number of variables, Rep. Math. Phys. 14 (1978), no. 3, 325-350.

[12] Yu. G. Kondratiev, P. Leukert, J. Potthoff, L. Streit, and W. Westerkamp, Generalized functionals in gaussian spaces: the characterization theorem revisited, J. Funct. Anal. 141 (1996), no. 2, 301-318.

[13] N. V. Krylov, An analytic approach to SPDEs, in Stochastic Partial Differential Equations: Six Perspectives, Math. Surveys Monogr. 64, R. Carmona and B. Rozovskii, eds., AMS, Providence, RI, 1999, pp. 185-242.

[14] N. V. Krylov, Introduction to the theory of diffusion processes, American Mathematical Society, Providence, RI, 1995. 
[15] N. V. Krylov and B. L. Rozovskii, Stochastic Evolution Equations, J. Sov. Math. 16 (1981), no. $4,1233-1276$

[16] J.-L. Lions, Quelques méthodes de résolution des problèmes aux limites non linéaires, Dunod, Paris, 1969.

[17] J.-L. Lions and E. Magenes, Problémes aux limites non homogènes et applications, volume 1, Dunod, Paris, 1968.

[18] S. V. Lototsky and B. L. Rozovskii, Stochastic differential equations: a Wiener chaos approach, From stochastic calculus to mathematical finance: the Shiryaev festschrift (Yu. Kabanov, R. Liptser, and J. Stoyanov, eds.), Springer, 2006, pp. 433-507.

[19] W Wiener chaos solutions of linear stochastic evolution equations, Ann. Probab. 34 (2006), no. 2, 638-662.

[20] _ Passive Scalar Equation in a Turbulent Incompressible Gaussian Velocity Field Russian. Math. Surveys. 59 (2004), No.2, 297-312

[21] R. Mikulevicius, B. L. Rozovskii, Linear parabolic stochastic PDE's and Wiener chaos. SIAM J. Math. Anal. 29, no. 2 (1998): 452-480.

[22] T. Martínez, M. Sanz-Solé, A lattice scheme for stochastic partial differential equations of elliptic type in dimension $n \geq 4$. Appl. Math. Optim. 54 (2006), no. 3, 343-368.

[23] D. Nualart, Applications of Malliavin Calculus to Stochastic Partial Differential Equations, Preprint.

[24] D. Nualart, Malliavin calculus and related topics, 2nd edition, Springer, New York, 2006.

[25] D. Nualart and B.L. Rozovskii, Weighted stochastic Sobolev spaces and bilinear SPDE's driven by space-time white noise, J. Funct. Anal. 149 (1997), no. 1, 200-225.

[26] D. Nualart and S. Tindel, Quazilinear stochastic elliptic equations with reflection, Stochastic processes and Applications, 57 (1995) 73-82.

[27] E. Pardoux, Equations aux derives partielles stochastiques non lineaires monotones. Etude de solutions fortes de type Ito, (1975). Thése Doct. Sci. Math. Univ. Paris Sud.

[28] B. L. Rozovskii, Stochastic evolution systems, Kluwer Academic Publishers, Dordrecht, 1990.

[29] J. B. Walsh, An introduction to stochastic partial differential equations, in Ecole d'Etè de Probabilités de Saint-Flour XIV 1984, 265-439 Lecture Notes in Math. 1180, Springer-Verlag, NewYork, 1986,

[30] M. Zakai, On the optimal filtering of diffusion processes, Ztschr. Wahrscheinlichkeitstheor und verw. Geb., 11 (1969), 230-243.

\section{Appendix.}

\section{A FACTORIAL INEQUALITY.}

Lemma A.1. For every multi-index $\alpha \in \mathcal{J}$,

$$
|\alpha| ! \leq \alpha !(2 \mathbb{N})^{2 \alpha}
$$

Proof. Recall that, for $\alpha=\left(\alpha_{1}, \ldots, \alpha_{k}\right) \in \mathcal{J}$,

$$
|\alpha|=\sum_{\ell=1}^{k} \alpha_{\ell}, \alpha !=\prod_{\ell=1}^{k} \alpha_{\ell} !, \mathbb{N}^{\alpha}=\prod_{\ell=1}^{k} \ell^{\alpha_{\ell}} .
$$

It is therefore clear that, if $|\alpha|=n$, then it is enough to establish (A1) for $\alpha$ with $\alpha_{k}=0$ for $k \geq n+1$, because a shift of a multi-index entry to the right increases the right-hand side of (A1) but does not change the left-had side. For example, if $\alpha=(1,3,2,0, \ldots)$ and $\beta=(1,3,0,2,0, \ldots)$, then $|\alpha|=|\beta|, \alpha !=\beta$ !, but $\mathbb{N}^{\alpha}<\mathbb{N}^{b e t a}$. Then

$$
4^{n} \geq\left(1+\frac{1}{2^{2}}+\ldots+\frac{1}{n^{2}}\right)^{n}=\sum_{\alpha_{1}+\ldots+\alpha_{n}=n} \frac{|\alpha| !}{\alpha !} \frac{1}{\mathbb{N}^{2 \alpha}}
$$


where the equality follows by the multinomial formula. Since all the term in the sum are non-negative, we get (A2).

The proof shows that inequality (A2) can be improved by observing that $\sum_{k \geq 1} k^{-2}=$ $\pi^{2} / 6<2$. One can also consider $\sum_{k \geq 1} k^{-q}$ for some $1<q<2$.

A VERsion OF THE TOEPLITZ LEMMA.

Lemma A.2. Assume that $f=f(t)$ is an integrable function and $\lim _{t \rightarrow+\infty}|f(t)|=0$. Then, for every $c>0, \lim _{t \rightarrow+\infty} \int_{0}^{t} e^{-c(t-s)} f(s) d s=0$.

Proof. Given $\varepsilon>0$, choose $T$ so that $|f(t)|<\varepsilon$ for all $t>T$. Then $\left|\int_{0}^{t} e^{-c(t-s)} f(s) d s\right| \leq e^{-c t} \int_{0}^{T} e^{c s}|f(s)| d s+\varepsilon \int_{T}^{t} e^{-c(t-s)} d s$. Passing to the limit as $t \rightarrow+\infty$, we find $\lim _{t \rightarrow+\infty}\left|\int_{0}^{t} e^{-c(t-s)} f(s) d s\right| \leq \varepsilon / c$, which completes the proof.

Current address, S. V. Lototsky: Department of Mathematics, USC, Los Angeles, CA 90089

E-mail address, S. V. Lototsky: lototsky@math.usc.edu

$U R L:$ http://math.usc.edu/ lototsky

Current address, B. L. Rozovskii: Division of Applied Mathematics, Brown University, Providence, RI 02912

E-mail address, B. L. Rozovskii: rozovsky@dam.brown.edu

$U R L:$ http://www.dam.brown.edu/people/rozovsky.html 